\title{
Temporal evolution of short-lived penumbral microjets
}

\author{
A. L. Siu-Tapia ${ }^{1}$, L. R. Bellot Rubio ${ }^{1}$, D. Orozco Suárez ${ }^{1}$, and R. Gafeira ${ }^{1,2}$ \\ 1 Instituto de Astrofísica de Andalucía (IAA-CSIC), Apdo. 3004, 18080 Granada, Spain \\ e-mail: siu@iaa.es; azaymisiu@gmail.com \\ ${ }^{2}$ Univ Coimbra, CITEUC - Center for Earth and Space Research of the University of Coimbra, Geophysical and Astronomical \\ Observatory, Coimbra 3040-004, Portugal
}

Received 7 May 2020 / Accepted 28 July 2020

\begin{abstract}
Context. Penumbral microjets (PMJs) is the name given to elongated jet-like brightenings observed in the chromosphere above sunspot penumbrae. They are transient events that last from a few seconds to several minutes, and their origin is presumed to be related to magnetic reconnection processes. Previous studies have mainly focused on their morphological and spectral characteristics, and more recently on their spectropolarimetric signals during the maximum brightness stage. Studies addressing the temporal evolution of PMJs have also been carried out, but they are based on spatial and spectral time variations only.

Aims. Here we investigate, for the first time, the temporal evolution of the polarization signals produced by short-lived PMJs (lifetimes $<2 \mathrm{~min}$ ) to infer how the magnetic field vector evolves in the upper photosphere and mid-chromosphere.

Methods. We use fast-cadence spectropolarimetric observations of the Ca II $854.2 \mathrm{~nm}$ line taken with the CRisp Imaging Spectropolarimeter at the Swedish $1 \mathrm{~m}$ Solar Telescope. The weak-field approximation (WFA) is used to estimate the strength and inclination of the magnetic field vector. By separating the Ca II $854.2 \mathrm{~nm}$ line into two different wavelength domains to account for the chromospheric origin of the line core and the photospheric contribution to the wings, we infer the height variation of the magnetic field vector.

Results. The WFA reveals larger magnetic field changes in the upper photosphere than in the chromosphere during the PMJ maximum brightness stage. In the photosphere, the magnetic field inclination and strength undergo a transient increase for most PMJs, but in $25 \%$ of the cases the field strength decreases during the brightening. In the chromosphere, the magnetic field tends to be slightly stronger during the PMJs.

Conclusions. The propagation of compressive perturbation fronts followed by a rarefaction phase in the aftershock region may explain the observed behavior of the magnetic field vector. The fact that such behavior varies among the analyzed PMJs could be a consequence of the limited temporal resolution of the observations and the fast-evolving nature of the PMJs.
\end{abstract}

Key words. sunspots - Sun: chromosphere - Sun: magnetic fields

\section{Introduction}

Penumbral microjets (PMJs) were among the first discoveries made with the Solar Optical Telescope (SOT; Tsuneta et al. 2008) aboard the Hinode spacecraft (Kosugi et al. 2007). Katsukawa et al. (2007) observed the ubiquitous occurrence of small-scale elongated jet-like brightenings above a sunspot penumbra in $\mathrm{Ca}$ II $\mathrm{H}$ images taken with the Broadband Filter Imager. The PMJ brightenings were characterized as short transients about $10-20 \%$ brighter than the surrounding environment, with lifetimes of $\sim 1 \mathrm{~min}$. They appear suddenly in image sequences, with apparent horizontal speeds on the order of $100 \mathrm{~km} \mathrm{~s}^{-1}$, and then fade gradually.

Katsukawa et al. (2007) also found that PMJs have typical lengths between 1000 and $4000 \mathrm{~km}$ (but up to $10000 \mathrm{~km}$ ) and widths around $400 \mathrm{~km}$, and that they appear to be well aligned with the superpenumbral fibrils. The brightenings are more easily identified when the sunspot is located closer to the limb, given that they make a larger angle with the bright photospheric penumbral filaments. Near the disk center, the PMJ brightenings are closely aligned with the bright penumbral filaments and so running-difference images are advantageous to detect them there. The center-to-limb variation of the PMJ orientation with respect to the penumbral filaments is due to the expansion of the magnetic field with height, as pointed out by Jurcák \& Katsukawa (2008). This interpretation can also explain the dependence of the PMJ inclination on the position within the penumbra (Jurcák \& Katsukawa 2008).

Magnetic reconnection between differently inclined field lines has been suggested as a possible driver of PMJs (e.g., Katsukawa et al. 2007; Katsukawa \& Jurcăk 2010; Jurcák \& Katsukawa 2010), given that the complex configuration of the magnetic field in sunspot penumbrae involves strong variations of the field strength and inclination over small spatial scales (e.g., Borrero \& Ichimoto 2011; Rempel \& Schlichenmaier 2011). This scenario has been supported by magnetohydrodynamic simulations (Magara 2010). In particular, Katsukawa \& Jurcăk (2010) and Jurcák \& Katsukawa (2010) studied the photospheric response associated with PMJs and found some downflows in the lower photosphere to be related to chromospheric brightenings. They claimed that these could be a possible signature of magnetic reconnection in the middle or low photosphere.

Tiwari et al. (2016) also report small-scale photospheric downflows and opposite polarity patches related to PMJs, while Vissers et al. (2015) find that some PMJs display transition region temperatures. These features might be a byproduct of magnetic reconnection occurring in the low atmosphere. However, Samanta et al. (2017) suggest that reconnection might 
occur higher in the atmosphere, in the low corona or transition region, based on observations of inward-moving elongated bright dots that appear before and are superposed to some PMJs.

Reardon et al. (2013) performed the first spectroscopic analysis of PMJs using Ca II 8542 A intensity profiles from the Dunn Solar Telescope and co-temporal Hinode $\mathrm{Ca}$ II $\mathrm{H}$ imaging data. They find a wide variety of temporal, spatial, and spectral behaviors among penumbral brightenings observed in the chromosphere, and argue that different physical processes could drive these transients. However, their temporal light curves in integrated intensity are very similar. They also found several events whose characteristics matched well the lifetimes and orientations of PMJs described in works based on SOT Ca II H filtergrams only. Such events were characterized by clear Ca II 8542 line wing emissions and almost unchanged line cores, similar to the spectral signatures of Ellerman bombs (e.g., Vissers et al. 2013). Reardon et al. (2013) also reported the presence of "precursors" around $1 \mathrm{~min}$ before the rapid impulsive brightening of some PMJs, which could be the signature of a shock, as proposed by Ryutova et al. (2008) in their bow-shock model.

The first statistical analysis of PMJ properties was performed by Drews \& Rouppe van der Voort (2017) by means of an automatic detection method using Ca II $8542 \AA$ spectroscopic data and $\mathrm{Ca}$ II $\mathrm{H}$ filtergrams taken at the Swedish $1 \mathrm{~m}$ Solar Telescope (SST). Their algorithm was set to select those PMJs whose spectral characteristics resemble Ellerman bombs in $\mathrm{H} \alpha$, such as enhanced line wings and an almost undisturbed line core, finding that the wing enhancements could extend to about $0.6 \AA$ from the line core. Most PMJs displayed stronger enhancements in the inner blue wing compared to the red wings. The average lifetime of the 453 identified PMJs was $90 \mathrm{~s}$, for an upper cutoff value of $8 \mathrm{~min}$, but the distribution peaked at shorter durations.

Recently, Esteban Pozuelo et al. (2019) investigated the polarization signals of 37 PMJs with lifetimes between 1 and $6.5 \mathrm{~min}$, finding that they tend to appear in regions where the inclination of the photospheric penumbral magnetic field undergoes significant horizontal variations, such as at the interface between spines and intra-spines (e.g., Borrero \& Ichimoto 2011) or in the outer penumbra where the field lines bend over at the end of the filaments (see also Tiwari et al. 2018). However, no strong Doppler shifts were observed and the estimated line-ofsight (LOS) motions had speeds lower than $4 \mathrm{~km} \mathrm{~s}^{-1}$, a value that is well below the apparent speeds on the order of $100 \mathrm{~km} \mathrm{~s}^{-1}$ previously inferred. Nonetheless, velocity gradients along the LOS were deduced from the shapes of the emission peaks of the Ca II $8542 \AA$ and Ca II K intensity profiles. The authors also find an increase in the temperature at chromospheric heights in their inversion results. Therefore, they suggest that PMJs could be a consequence of magnetic reconnection in the low photosphere, a process that might trigger an upwardly propagating perturbation responsible for heating the chromosphere. In addition, Esteban Pozuelo et al. (2019) find that PMJs exhibit enhanced polarization signals, particularly the Ca II $8542 \AA$ circular polarization profiles, which in some cases display extra lobes. The presence of extra lobes is explained by the authors to be a consequence of the typical moustache shapes of the intensity profiles, given that they can be reproduced using the weak field approximation (WFA).

In this work we investigate for the first time the temporal evolution of the polarization profiles of the most common PMJs, which, according to the statistical study by Drews \& Rouppe van der Voort (2017), are those with lifetimes under $2 \mathrm{~min}$. To our knowledge, the magnetic properties and evolution of these very short events have not been analyzed before. The reason is that such a study requires challenging observations (ultra-fast spectropolarimetry) due to their highly dynamic nature.

Studying the temporal changes in the magnetic field configuration and in the plasma properties occurring in and around PMJs can give us insight on their physical nature and on where they lie on the solar atmosphere. However, such an analysis faces various observational limitations. Because of the extremely short lifetimes of the most frequent PMJs, observations with the fastest temporal cadence possible are needed. This requirement puts restrictions on other observational parameters, such as the number of spectral lines that can be scanned, the width of the observed spectral range and the spectral sampling. Moreover, it implies short exposure times, which can substantially increase the noise.

Here, we approach this problem by making trade-offs between the different observational parameters as described in Sect. 2. In Sect. 3 we explain our identification criteria and the diagnostic tools we use. In Sect. 4 we present individual examples and statistical results, which are summarized in Sect. 5. Finally, in Sect. 6 we discuss our findings and draw conclusions.

\section{Observations}

Spectropolarimetric observations of the main sunspot in active region 12553 were carried out on 2016 June 16 at the SST (Scharmer et al. 2003) between 08:06:00 and 09:27:53 UT using the CRisp Imaging Spectrometer (CRISP; Scharmer et al. 2008). The sunspot displayed positive magnetic polarity and was observed very close to disk center, at a heliocentric angle of $\sim 8^{\circ}$ $\left(19^{\prime \prime} \mathrm{W}, 125^{\prime \prime} \mathrm{S}\right)$.

Our CRISP data consist of time series of full Stokes measurements in the chromospheric Ca II $8542 \AA$ line and in the photospheric Fe I $6173 \AA$ line. In order to perform observations with a temporal cadence fast enough to study the evolution of the shortest-duration PMJs, the Ca II line was sampled at nine wavelength positions in steps of $150 \mathrm{~m} \AA$, from -600 to $+600 \mathrm{~m} \AA$ around the line core, plus a point at $+2.4 \AA$. Similarly, the photospheric Fe I line was sampled at five wavelength positions in steps of $40 \mathrm{~m} \AA$, from -80 to $+80 \mathrm{~m} \AA$ around the line core, plus one position in the continuum at $+400 \mathrm{~m} \AA$. Sampling the two lines required $17 \mathrm{~s}$ ( $11 \mathrm{~s}$ for the $\mathrm{Ca}$ II line and $6 \mathrm{~s}$ for the Fe I line), using nine accumulations per modulation state and wavelength. The later was set as the best possible compromise between the total integration time and the photon noise level. The duration of the time series is $\sim 82 \mathrm{~min}$, that is, 290 time steps. The field of view (FOV) is $\sim 50^{\prime \prime} \times 50^{\prime \prime}$ with a pixel size of $0.05^{\prime \prime}$.

The data were reduced using the CRISPRED pipeline (de la Cruz Rodríguez et al. 2015) and reconstructed with the Multi-Object-Multi-Frame-Blind-Deconvolution technique (Löfdahl 2002; van Noort et al. 2005), separately for each line. Unfortunately, the reconstruction of the Fe I $6173 \AA$ line resulted in poor quality data and is therefore not used in this study. However, the Ca II $8542 \AA$ line has contributions from different layers as its line core originates from layers at heights between $\sim 800$ and $1000 \mathrm{~km}$, and the wings are formed at considerably deeper layers, mainly below $500 \mathrm{~km}$ (Cauzzi et al. 2008). Thus, some information about the photospheric properties of the sunspot is available from the Ca II $8542 \AA$ line wings and the wavelength point at $+2.4 \AA$.

In order to reduce the noise level of the observations, we have convolved the complete dataset with a three by three low-pass filter kernel so that each pixel in the resulting images has a value equal to the average value of its neighboring pixels in the original image. The noise levels in the resulting images are 


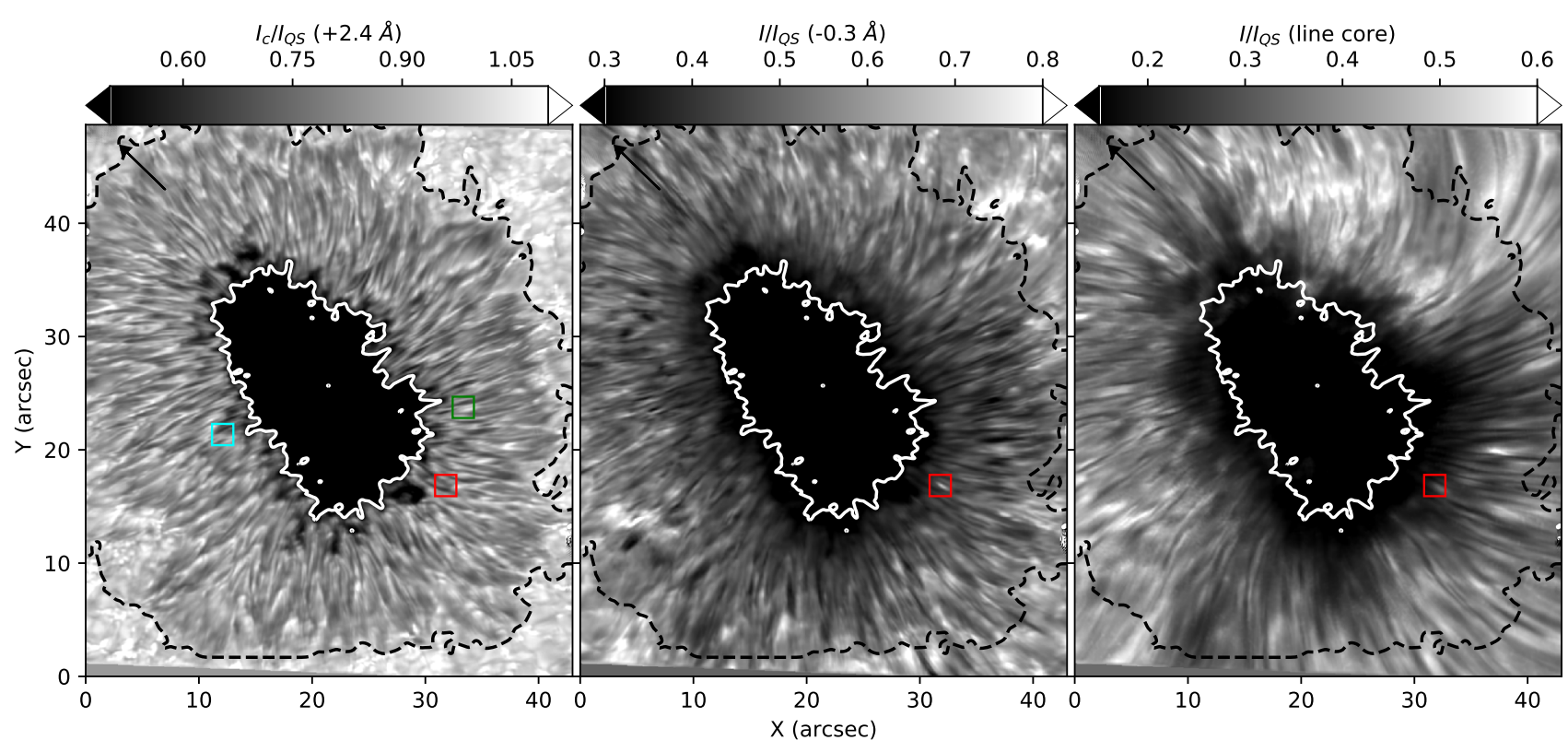

Fig. 1. Main sunspot in active region 12553 on 2016 June 16 recorded with the CRISP instrument at different wavelengths within the Ca II $8542 \AA$ line. Left panel: continuum intensity $I_{\mathrm{c}}$ image, at $+2.4 \AA$ from the line core. Central panel: blue-wing image, at $-0.3 \AA$ from the line core. Right panel: line core image. All the images are normalized to the mean continuum value in the quiet sun, $I_{\mathrm{QS}}$. The umbra-penumbra boundary (white contours) is at $I_{\mathrm{c}} / I_{\mathrm{QS}}=0.45$ and the outer penumbral boundary (black dashed contours) is at $I_{\mathrm{c}} / I_{\mathrm{QS}}=0.98$. Black arrows point toward the disk center. Small colored squares on the continuum image highlight the location of three PMJs referred to as PMJ 1 (red), PMJ 2 (green), and PMJ 3 (cyan) in the main text. The images correspond to the frame in which PMJ 1 displays the maximum blue-wing brightness.

$\sigma_{Q, U} \sim 3 \times 10^{-3}$, and $\sigma_{V} \sim 4 \times 10^{-3}$, as measured on the continuum wavelength maps. Finally, the data have been corrected for residual crosstalk by using the average value of the $\alpha_{s}$ maps at each time step, where $\alpha_{s}=s\left(\lambda_{\mathrm{c}}\right) / I\left(\lambda_{\mathrm{c}}\right)$ with $s=Q, U, V$ and $\lambda_{\mathrm{c}}$ corresponding to the continuum wavelength.

Figure 1 displays the general appearance of the observed sunspot at different wavelengths within the Ca II $8542 \AA$ line: continuum (left), blue wing (central), and line core (right panel). The image on the right shows that there is a predominantly brighter region in the northern part of the sunspot where the line core is in emission. This region maintains line core emissions during our complete time series and makes the detection of PMJs very difficult in that part of the penumbra.

\section{Analysis}

\subsection{PMJ identification}

We are interested in studying the most common PMJs which, according to Drews \& Rouppe van der Voort (2017), are the events with lifetimes shorter than $2 \mathrm{~min}$. In particular, the polarimetric properties of the shortest duration PMJs ( $<1 \mathrm{~min}$ ) have never been analyzed, inasmuch as the only available study focused on PMJs that live longer than $1 \mathrm{~min}$ (Esteban Pozuelo et al. 2019).

Given that PMJs show a preference for larger brightness enhancements in the blue wing of the Ca II $8542 \AA$ intensity profile, with the help of the temporal sequence of images at $-0.3 \AA$ from the line core and of the running difference images (intensity differences with respect to the frame recorded $17 \mathrm{~s}$ before), we identified 36 PMJs with lifetimes shorter than 2 min through visual inspection. This means that the associated brightening is observed during a maximum of seven frames $(119 \mathrm{~s})$ and a minimum of one frame $(17 \mathrm{~s})$ in the blue wing images and/or in their running differences. The brightenings must display elon- gated shapes and intensity changes in the blue wing (at $-0.3 \AA$ ) of at least $10 \%$ of their intensity profiles averaged over the full time sequence.

Figure 2 shows the location and lifetimes of the 36 shortlived PMJs identified in our complete temporal sequence. The spatial distribution reveals a larger number of PMJs occurring in the limb-side penumbra. However, this trend could be the result of intrinsic asymmetries of this particular sunspot, such as the persistent line core emissions detected over the centerside penumbra which hinder the identification of possible wing emissions produced by PMJs in that region.

The PMJs were observed at different radial positions within the penumbra, mainly above the interface between dark and bright filaments (13 cases), but some PMJs occurred at the end of bright penumbral filaments (six cases) or above the filaments (six cases), and between tails and heads of different filaments (nine cases). Two PMJs emerged just at the outer penumbral boundary. The lifetime distribution of the 36 PMJs has a mean value $\mu=71 \mathrm{~s}$ and a median $m=68$ s (i.e., four frames).

A total of 19 PMJs (circle symbols in Fig. 2) displayed clear brightness enhancements in the line core within regions that are slightly shifted in the radial direction from the PMJ regions, as shown in the following subsection. The observed displacements are interpreted as a projection effect of the chromospheric counterparts (CCs) of magnetic field lines that connect with the PMJ regions at photospheric layers.

\subsection{Spectral and polarimetric characteristics}

An example of a short-lived PMJ which is visible during less than $1 \mathrm{~min}$, hereafter referred to as PMJ 1, is displayed in Fig. 3. The figure shows images of the continuum intensity (left column), the blue-wing intensity at $-0.3 \AA$ (central column), and the normalized running differences of the blue-wing images for 

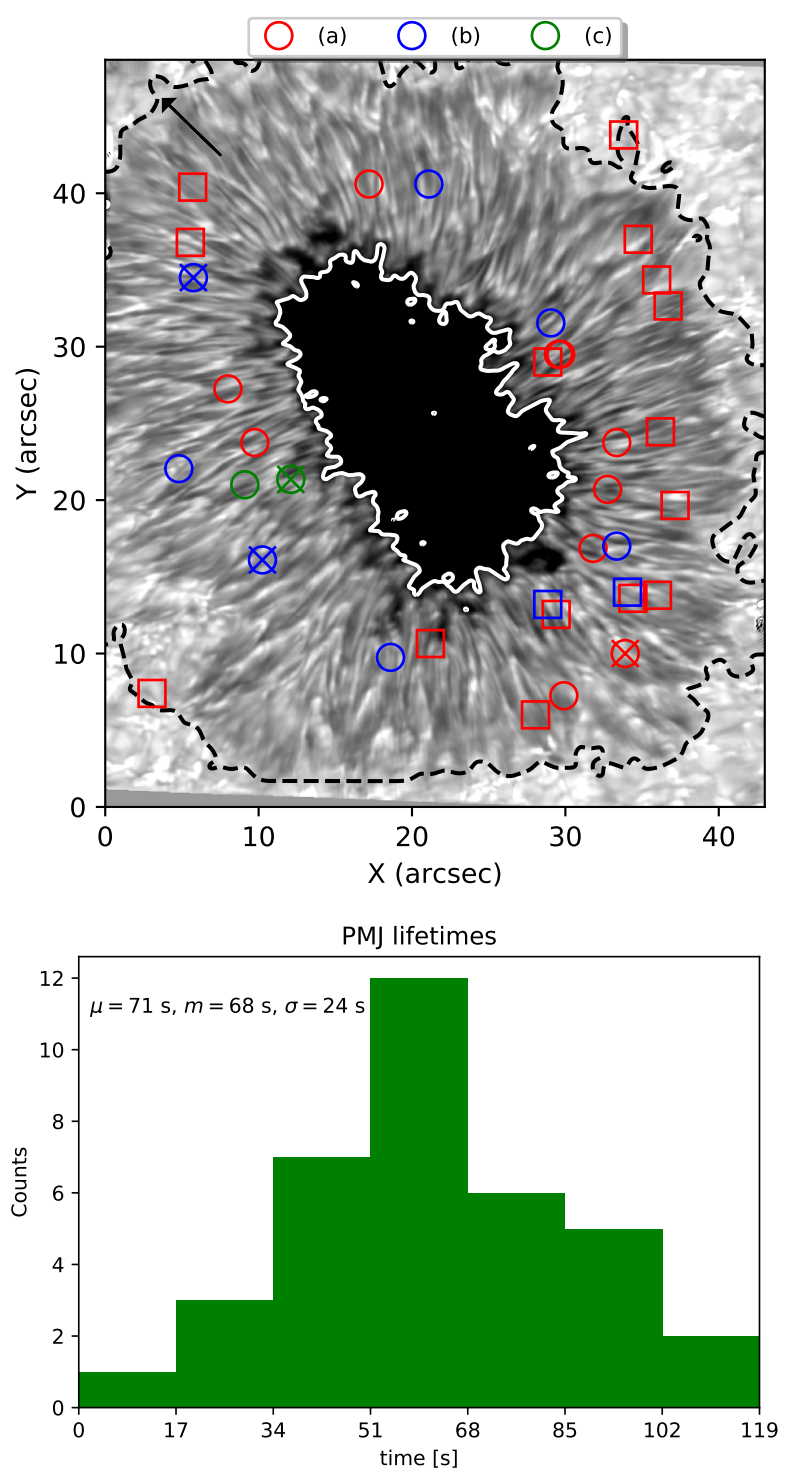

Fig. 2. Top: continuum intensity image of the sunspot in the same format as Fig. 1, displaying the location of the 36 short-lived PMJs that were identified in the complete temporal sequence, with lifetimes shorter than 2 min. The different colors of the markers indicate different types of evolution of the photospheric magnetic field as described in the main text. PMJs displaying line core brightness enhancements larger than $10 \%$ are shown with circles; otherwise, they are indicated with squares. The crossed symbols stand for PMJs displaying clear changes of the chromospheric magnetic field during their brightening. Bottom: lifetime distribution for the selected PMJs. The mean $\mu$, median $m$, and standard deviation $\sigma$ of the distribution are indicated in the upper left corner.

five consecutive frames (right column). The PMJ brightening is visible in three frames (from second to fourth row) in the bluewing images, so that its estimated lifetime is $51 \mathrm{~s}$. The intensity change in the blue wing is larger than $10 \%$ between the first and the second frame, and it is nearly $36 \%$ between the first and the maximum brightness frame (third row). The intensity change is negative after the maximum brightness stage (fourth row). We refer to the frame preceding the first appearance of the PMJ brightening as "before" (frame in first row of Fig. 3), and to the frame after the PMJ brightening vanishes as "after" (frame in fifth row of Fig. 3).
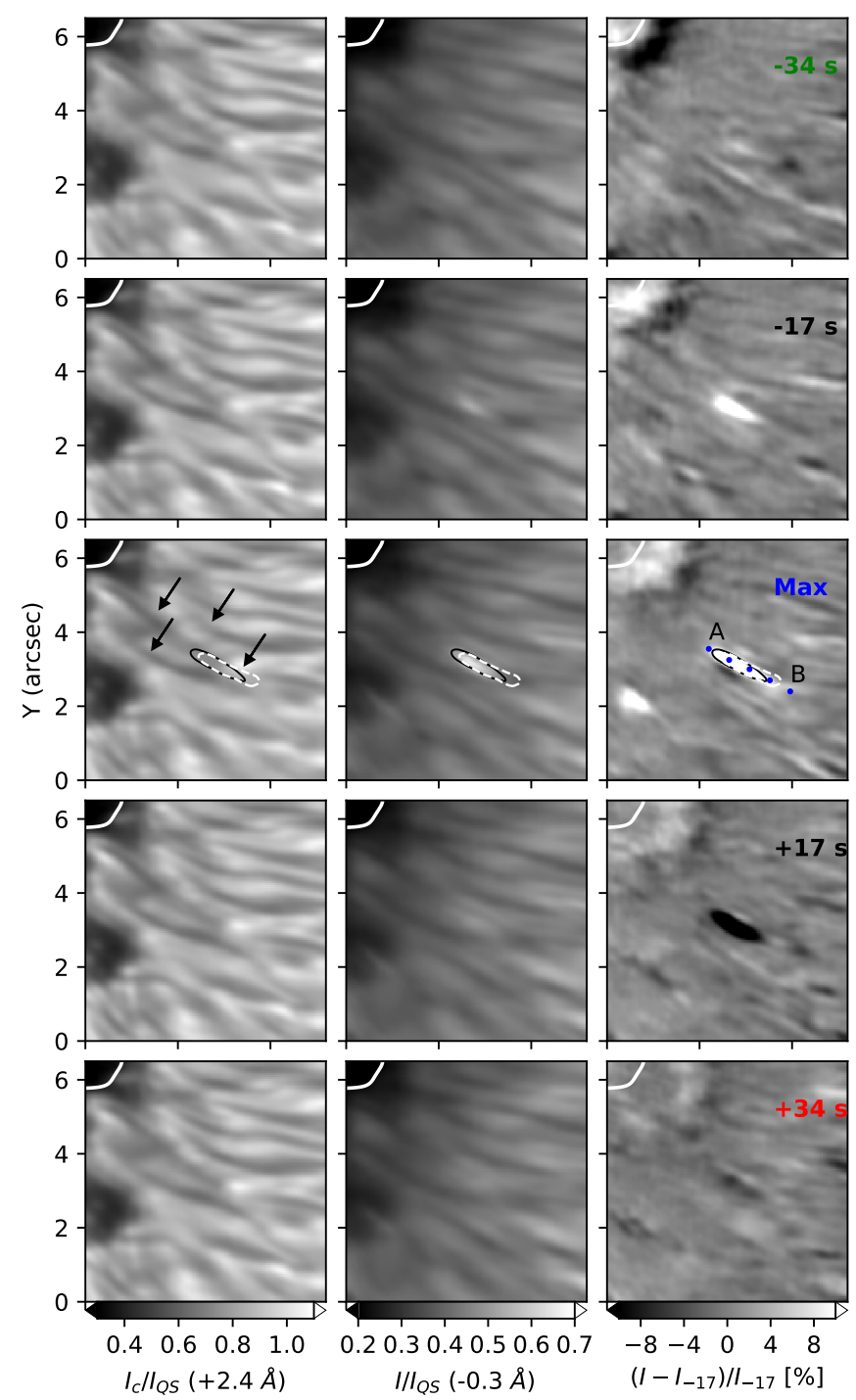

Fig. 3. Example of the evolution of a PMJ whose brightening is observed during three frames (lifetime of $51 \mathrm{~s}$ ) in the inner limb-side penumbra (labeled as PMJ 1 in Fig. 1). Left: continuum intensity images at $+2.4 \AA$ from the line core. Center: blue-wing images, at $-0.3 \AA$ from the core. Right: blue-wing intensity difference images with respect to the frame recorded $17 \mathrm{~s}$ before. Time increases from top to bottom, see labels on the right panels. Middle row: maximum brightness stage. Black contours delimit the PMJ area, that is, the region displaying bluewing brightness enhancements larger than $10 \%$. White dashed contours enclose the $\mathrm{CC}$, defined as the region displaying a line-core brightness enhancement larger than $10 \%$. White solid lines delimit the inner penumbral boundary. Black arrows indicate three penumbral filaments and a bright grain observed in the continuum images near the brightening region.

The PMJ brightening is not visible in the continuum images. The black elongated contours in the plots enclose the PMJ during the maximum brightness stage. Three penumbral filaments with dark lanes meet in such region. The PMJ brightening occurs near the end (or tail) of the central filament, which protrudes from the inner penumbral boundary into the umbra. In addition, a bright penumbral grain (which could be either the head of a fourth filament or a supersonic downflow at the end of one of the filaments, see Esteban Pozuelo et al. 2016) is also visible in the radial direction further away from the tail of the central filament. 

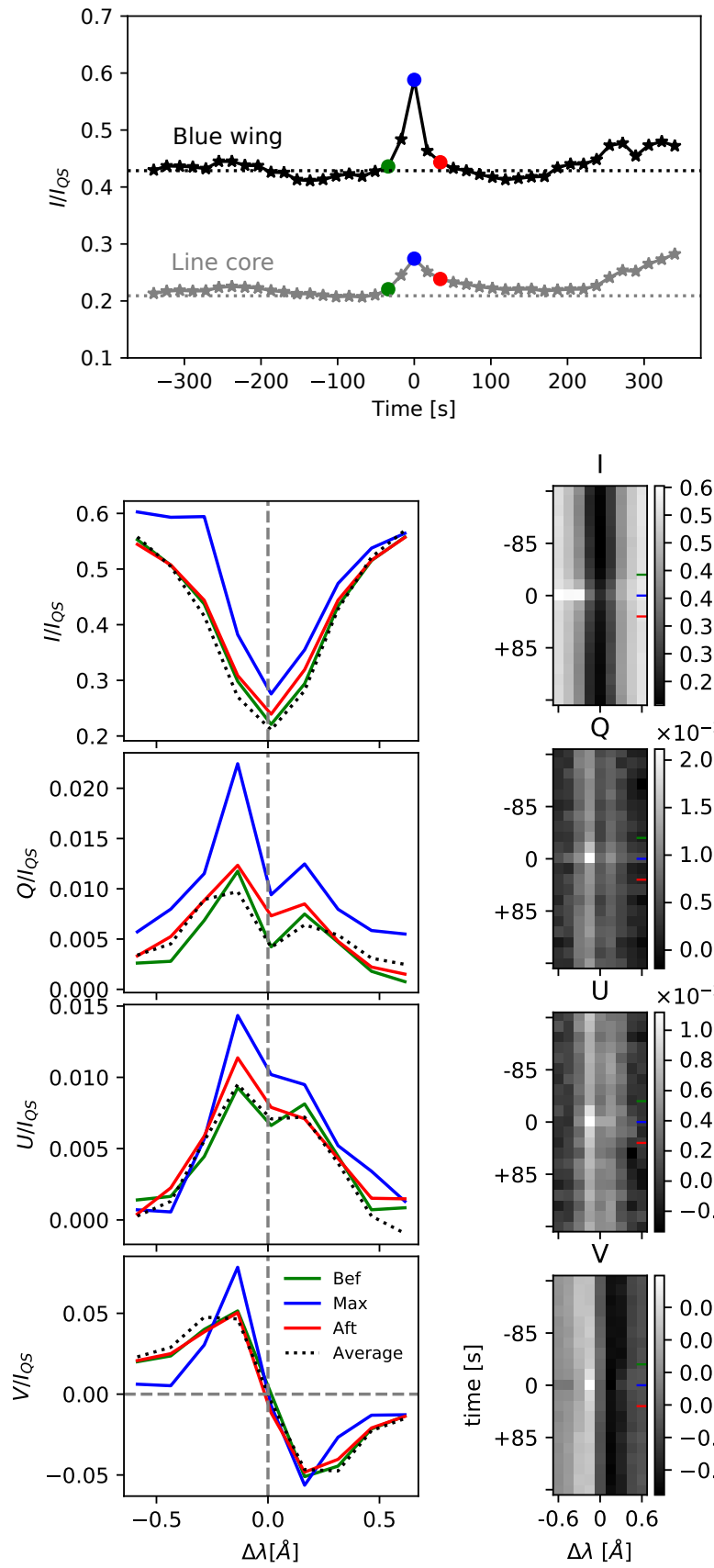
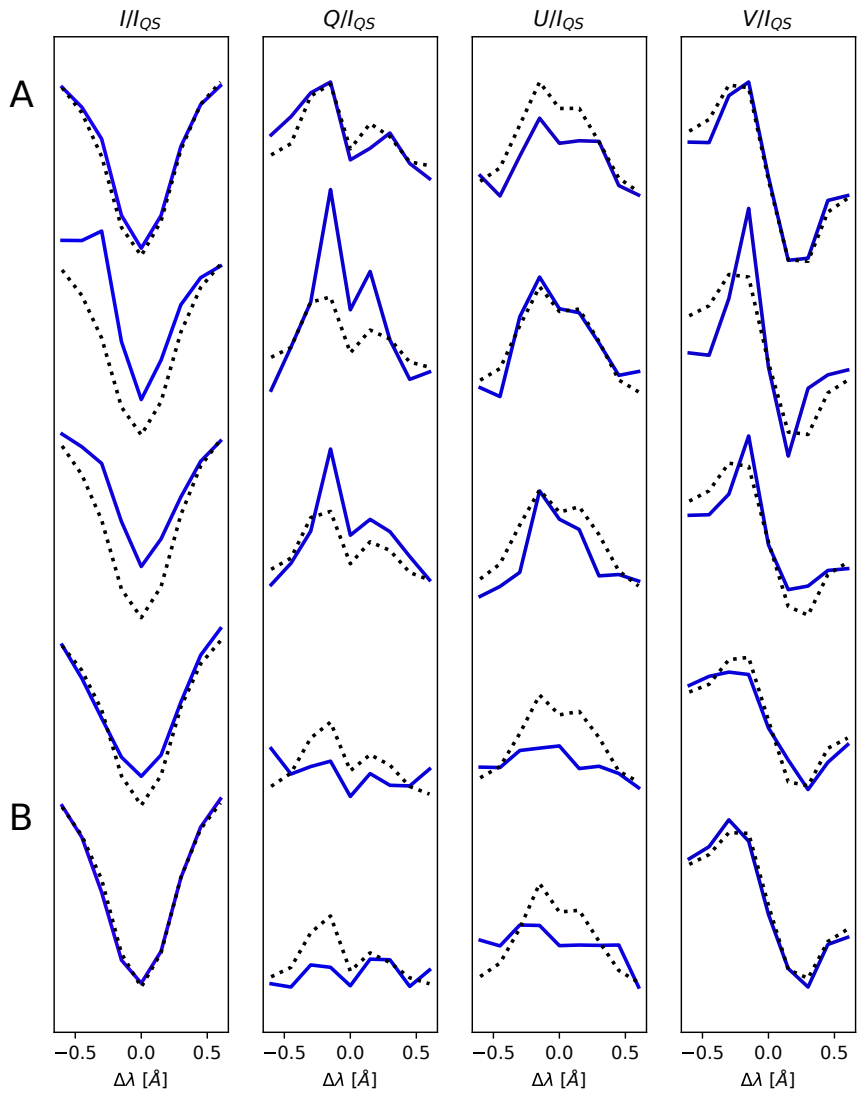

Fig. 5. Spatial variation of the Stokes profiles along PMJ 1. The profiles emerged from the locations marked with blue symbols in Fig. 3, from point A (top) to point B (bottom). The solid profiles correspond to the maximum brightness stage and the dotted profiles to the temporal averages over the complete time series at the same locations.

pixel (MBP), that is to say, averaged over its eight closest neighbors. These pixels display brightness fluctuations before and after the PMJ, with amplitudes that are up to $4 \%$ of their mean intensity over the full time sequence (horizontal dotted lines). Moreover, about $200 \mathrm{~s}$ after the maximum brightness stage, these pixels present a generally enhanced brightness of around $8 \%$ compared with their average over the full time sequence. However, the PMJ brightening stands well above such enhanced intensity, the noise level and other brightness fluctuations that might be caused by, for example, Doppler shifts of the line, waves, bad seeing conditions, etc. Also, the PMJ occurs abruptly while other brightness fluctuations vary more smoothly, which allows for a precise identification of PMJs. Notably, the line-core intensity remains slightly enhanced after the PMJ disappears in the blue wing wavelengths.

Figure 4 also shows the polarization signals of the Ca II 8542 A line as observed in the MBP during the three stages of interest (colored solid lines in the left panels) as well as the Stokes profiles at the MBP averaged over the complete time sequence (black dotted lines). The Stokes profiles observed during the stages before (green) and after (red) are remarkably similar to the temporally averaged profiles, but display slightly narrower intensity profiles as well as marginally brighter line cores.

At the maximum brightness stage (blue), all four Stokes profiles display enhanced signals. In particular, the intensity profile shows an emission in the blue wing, which peaks near $\Delta \lambda=$ $-0.3 \AA$. The line core and red wing intensities are also enhanced, top panel of Fig. 4 shows the temporal evoluti core intensity (gray) in the vicinity of the maximum brightness 
but their increase is considerably smaller. The circular and linear polarization profiles display larger signals at the maximum brightness stage, particularly in the blue wing. However, Stokes $V$ decreases toward the outer wings. The wavelength with maximum polarization signal in Stokes $Q, U$, and $V$ occurs around $\Delta \lambda=-0.15 \AA$. Such enhanced polarization signals produced by the PMJ are also clearly distinguishable from the spectrotemporal plots (right panels). In particular, both Stokes $I$ and $V$ stand well above the noise level, whilst enhanced Stokes $Q$ and $U$ signals appear on a noisier background but with amplitudes that are still above $3 \sigma$ at the maximum brightness stage.

In Fig. 5, we show the Stokes profiles emerging from selected pixels along the PMJ, indicated by the blue symbols (from point A to B) in Fig. 3. The first set of profiles, which emerged from a pixel located outside the PMJ contour (point A), display regular shapes and are very similar to their temporally averaged profiles. The next set of profiles emerged from the PMJ region and show enhanced intensity as well as larger polarization signals in the blue wing wavelengths. As we keep moving radially outward, the blue-wing intensity enhancements gradually decrease and those in the line core wavelengths become more notorious, reaching a point where the profiles only show an enhancement in the core but not in the blue wing. The profiles have more regular shapes outside the $\mathrm{CC}$ region (near point B).

The radial shift between the PMJ and CC regions is roughly 12 pixels or $\sim 500 \mathrm{~km}$, since our pixel size corresponds to $\sim 41 \mathrm{~km}$. It can be explained as the result of the three-dimensional structure of the magnetic field. Due to the expansion with height of the penumbral magnetic field, a given field line will be observed slightly displaced radially outward in the chromosphere with respect to the photosphere, which is what we detect in this case. The more inclined the field line, the larger the radial displacement with height. This suggests that the brightness enhancements detected in the line wing and the line core correspond to a perturbation or process that occurs on the same field lines at different heights in the atmosphere. The dependence of the intensity enhancements on wavelength and radial position during the microjet might be the result of plasma heating occurring along a penumbral structure, from the upper photosphere to the low chromosphere.

We also inspected the total linear polarization (TLP), total circular polarization (TCP), and net circular polarization (NCP) in the PMJ environment, defined as:

$$
\begin{aligned}
& \mathrm{TLP}=\int_{-0.6 \AA}^{+0.6 \AA} \sqrt{\frac{Q^{2}(\lambda)+U^{2}(\lambda)}{I_{\mathrm{QS}}^{2}}} \mathrm{~d} \lambda, \\
& \mathrm{TCP}=\int_{-0.6 \AA}^{+0.6 \AA} \frac{|V(\lambda)|}{I_{\mathrm{QS}}} \mathrm{d} \lambda, \\
& \mathrm{NCP}=\int_{-0.6 \AA}^{+0.6 \AA} \frac{V(\lambda)}{I_{\mathrm{QS}}} \mathrm{d} \lambda .
\end{aligned}
$$

Figure 6 shows the temporal evolution of these quantities for the three PMJs. As in all 36 PMJs in our sample, the TLP maps (transverse magnetograms) show enhanced signals inside the PMJ area (yellow contours). These enhanced signals generally reach a maximum at the maximum brightness stage. Moreover, in many cases, such as in PMJ 2 (middle panels) and PMJ 3 (bottom panels), some patches of positive TLP signal can be detected since the stage before within the yellow contour lines. Such preexisting features of enhanced linear polarization are observed in more than $60 \%$ of the cases, mainly inside the PMJ area but also in regions adjacent to it, which might indicate the presence of an enhanced horizontal component of the magnetic field in the pre-PMJ phase.

The TCP signals display different behaviors among the 36 PMJs. For PMJ 1, as in most of the cases $(69 \%$ of the total sample), the TCP undergoes a slight decrease at the maximum brightness stage, despite the fact that the Stokes $V$ profiles display enhanced signals in their inner wings. However, the outer wings show a notable signal reduction at maximum (see Fig. 4 for PMJ 1) which explains why the TCP decreases in these cases. In contrast, the TCP in PMJ 2 displays no clear changes. This is the behavior observed in four PMJs from our sample. Finally, as in PMJ 3, a total of seven PMJs displayed an increase inside the microjet region at maximum with respect to the stage before. This indicates that there are different types of evolution in the longitudinal component of the magnetic field for each case, which will be further investigated in the following sections.

The NCP is a measure of how asymmetric the Stokes $V$ profiles are. It can be used as a proxy for gradients along the LOS of the magnetic field and of the LOS velocities. We found an increase in the NCP inside the PMJ regions in all 36 cases. The NCP values are generally positive inside the PMJs and reach a maximum value at the maximum brightness stage, such as in PMJ 2. However, there are also a few cases in which the maximum NCP occurs one frame before or after the maximum brightness phase, such as in PMJs 1 and 3.

\subsection{Magnetic field configuration}

To investigate how the magnetic field configuration evolves during PMJs, we consider the weak-field approximation (WFA). The WFA can be applied when the thermal broadening $\Delta \lambda_{D}$ of the line is much larger than the Zeeman splitting $\Delta \lambda_{B}$ (e.g., Landi Degl'innocenti \& Landolfi 2004). Under the assumption of a constant and sufficiently weak magnetic field in the region of the solar atmosphere where the spectral line is formed, the radiative transfer equation (RTE) can be solved analytically by considering small perturbations of the zero-field case. In such case, the analytical solution is the WFA and the first order perturbation (in which Stokes $Q$ and $U$ are zero) yields the following expression:

$V=-\Delta \lambda_{B} f g \cos (\gamma) \frac{\partial I}{\partial \lambda}=\alpha f B_{\mathrm{LOS}} \frac{\partial I}{\partial \lambda}$,

where $g$ is the effective Lande factor of the line, $f$ is the filling factor which represents the fraction of the resolution element covered by the magnetic field, and $\gamma$ is the inclination of the magnetic field vector with respect to the LOS.

Expression (4) implies that Stokes $V$ is proportional to the partial derivative of Stokes $I$ with respect to wavelength, and that the factor of proportionality depends on the longitudinal component of the magnetic field, $B_{\mathrm{LOS}}=B \cos (\gamma)$. Therefore, $B_{\mathrm{LOS}}$ can be estimated by applying a linear least-squares fit to Eq. (4), which yields:

$B_{\mathrm{LOS}}=\frac{\sum_{i} \frac{\partial I\left(\lambda_{i}\right)}{\partial \lambda_{i}} V\left(\lambda_{i}\right)}{\alpha \sum_{i}\left(\frac{\partial I\left(\lambda_{i}\right)}{\partial \lambda_{i}}\right)^{2}}$,

with $\alpha=-4.67 \times 10^{-13} g \lambda_{0}^{2}$ (e.g., Martínez González \& Bellot Rubio 2009). In Eq. (5), we have set $f=1$ since we are in a sunspot.

Similarly, the expressions for the linear polarization (Stokes $Q$ and $U$ ) can be derived by perturbing the RTE to second order 

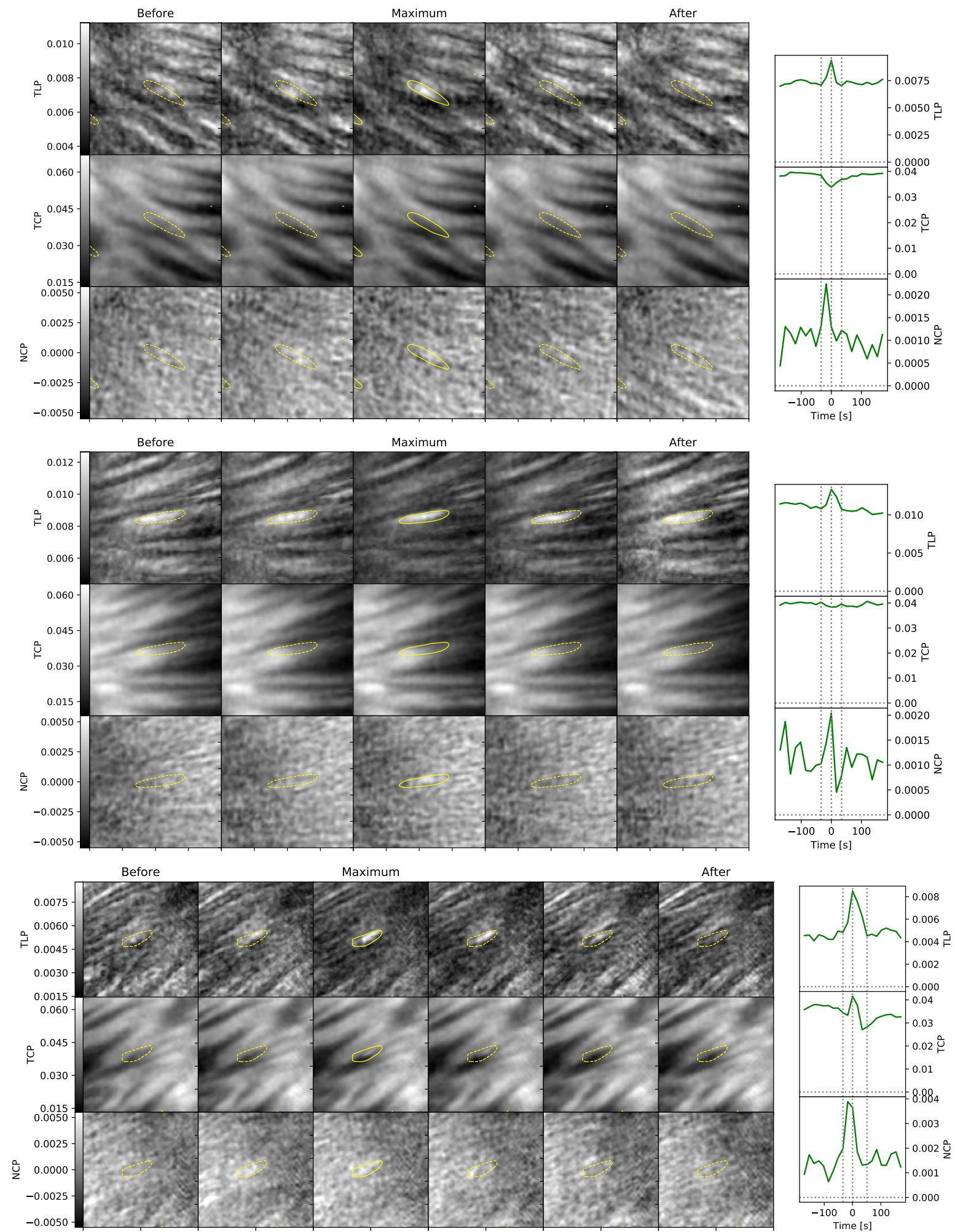

Fig. 6. Temporal evolution of three PMJs whose positions within the penumbra are indicated in Fig. 1: PMJ 1 (top), PMJ 2 (middle), and PMJ 3 (bottom). Right: for each case the panels show, from top to bottom, maps of total linear polarization (TLP), total circular polarization (TCP), and net circular polarization (NCP). Contours enclose the PMJ area. Time runs from left to right with $17 \mathrm{~s}$ cadence. Left: evolution of the average TLP, TCP, and NCP in the microjet area. The vertical dotted lines correspond to the frames before, maximum, and after.

(e.g., Landi Degl'innocenti \& Landolfi 2004). Such expressions involve the first and second derivatives of the intensity profile with respect to wavelength. Their combination with Eq. (4), along with the application of a least-squares minimization procedure, lead to the following relation for the inclination angle $\gamma$ :

$\tan ^{2}(\gamma)=\frac{4}{3} \frac{g^{2}}{G} \frac{\sum_{i}\left|\lambda_{i}\right|\left|L_{i}\right|\left|V_{i}\right|^{2}\left|\frac{\partial I}{\partial \lambda}\right|_{i}}{\sum_{i}\left|V_{i}\right|^{4}}$ 

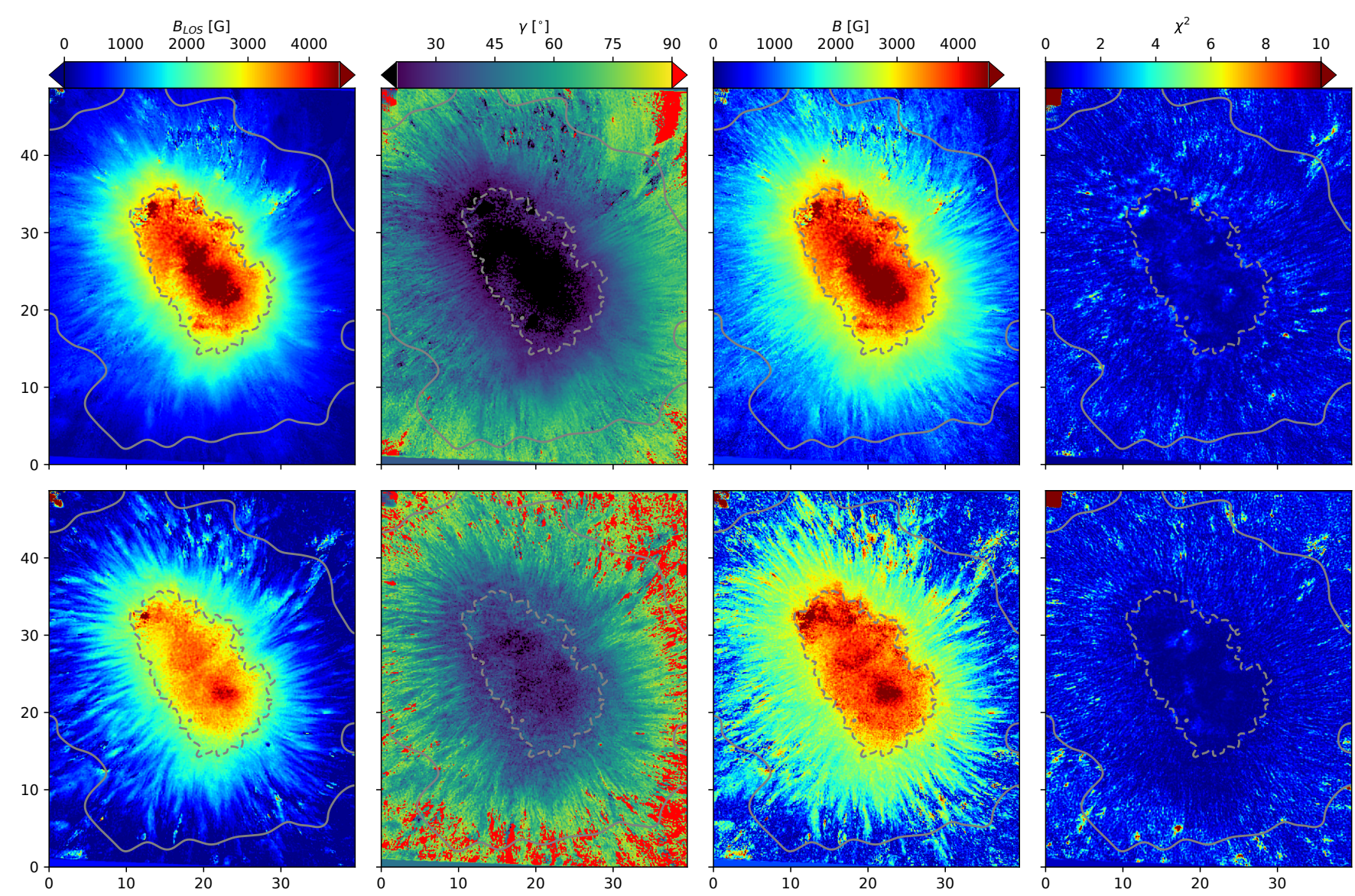

Fig. 7. From left to right: maps of the longitudinal component of the magnetic field $B_{\mathrm{LOS}}$, the field inclination $\gamma$ with respect to the LOS, the magnetic field strength $B$, and the merit function $\chi^{2}$ that result from the WFA applied to the line core wavelengths (top panels) and to the line wing wavelengths (bottom panels) for the entire sunspot as recorded at 08:13:22 UT. Red patches on the inclination maps are pixels where $\gamma>90^{\circ}$. Smoothed gray contours delimit the inner (dashed) and outer (solid) penumbral boundaries.

where $\left.L_{i}=\sqrt{(} Q_{i}^{2}+U_{i}^{2}\right)$ and $G$ is the Landé factor for the transverse magnetic field (see e.g., Hammar 2014). Equation (6) gives only the modulus of the inclination. The polarity of the field is determined by Eq. (5). Thus, the value of $\gamma$ (in degrees) can be defined as follows:

$\gamma= \begin{cases}|\gamma| & \text { if } B_{\mathrm{LOS}}>0 \\ 180^{\circ}-|\gamma| & \text { if } B_{\mathrm{LOS}}<0 .\end{cases}$

The validity of the WFA for Ca II $8542 \AA$ ( $g \approx 1.10$ and $G \approx 1.18$ ) has been studied by, among others, Hammar (2014) and Centeno (2018), who conclude that the WFA allows for an efficient and rapid inference of the chromospheric magnetic field vector from Ca II 8542 observations. The modest Landé factor and broad shape of this line guarantee the validity of the WFA even when the magnetic field is relatively strong.

Hammar (2014) and de la Cruz Rodríguez et al. (2013) estimated that, for a chromospheric model in which the temperature is $4500 \mathrm{~K}$ and the micro-turbulent velocity is $3 \mathrm{~km} \mathrm{~s}^{-1}$, the Ca II $8542 \AA$ line is in the weak-field regime for chromospheric fields weaker than $2500 \mathrm{G}$. However, the Doppler width and consequently the upper limit of the magnetic field both increase at higher temperatures. Therefore, the wings of the line can also be treated under the WFA for the stronger magnetic fields of the photosphere.

Specifically, Centeno (2018) modeled this line considering scenarios in which the field strength decreased with height and found that the WFA applied to the wings can be used to probe magnetic fields around $\log (\tau)=-1.4$ with remarkable accuracy for a range of field strengths of up to $4000 \mathrm{G}$. She also found that when evaluated in the line core $\left(\lambda_{0} \pm 250 \mathrm{~m} \AA\right.$, probing an average optical depth of $\log (\tau)=-5.3$ ) the WFA errors are only about $10 \%$ of the real value for field strengths up to $1200 \mathrm{G}$.

In Fig. 7, we display the result of the WFA applied to the entire sunspot as observed at 08:13:22 UT. The figure shows maps of the longitudinal component of the magnetic field $B_{\mathrm{LOS}}$, the inclination angle $\gamma$, the magnetic field strength $B=B_{\mathrm{LOS}} / \cos (\gamma)$, and the merit function $\chi^{2}$ which represents the sum of the squared differences between the observed Stokes $V$ profiles and the values resulting from the WFA, normalized to the noise level and number of wavelength points.

The WFA has been applied separately to the core of the line using the wavelength interval between $\pm 0.3 \AA$ (upper panels in Fig. 7) and to the wings using the wavelength points at $\Delta \lambda=$ $[-0.6,-0.45,0.45,0.6] \AA$ (lower panels). In general, Eq. (4) can reproduce the shape of the observed Stokes $V$ profiles, with most fits inside the spot having $\chi^{2}<3$ in both the wing and the line core regimes. However, the WFA returns magnetic fields larger than $2500 \mathrm{G}$ in the umbral areas. These values are above the field strength limit for the validity of the WFA in the chromosphere inferred by Hammar (2014) and, therefore, are not reliable.

In the rest of the sunspot, the well-known penumbral fine structure of the photospheric magnetic field is clearly discernible in the bottom maps, whilst a more extended magnetic canopy 

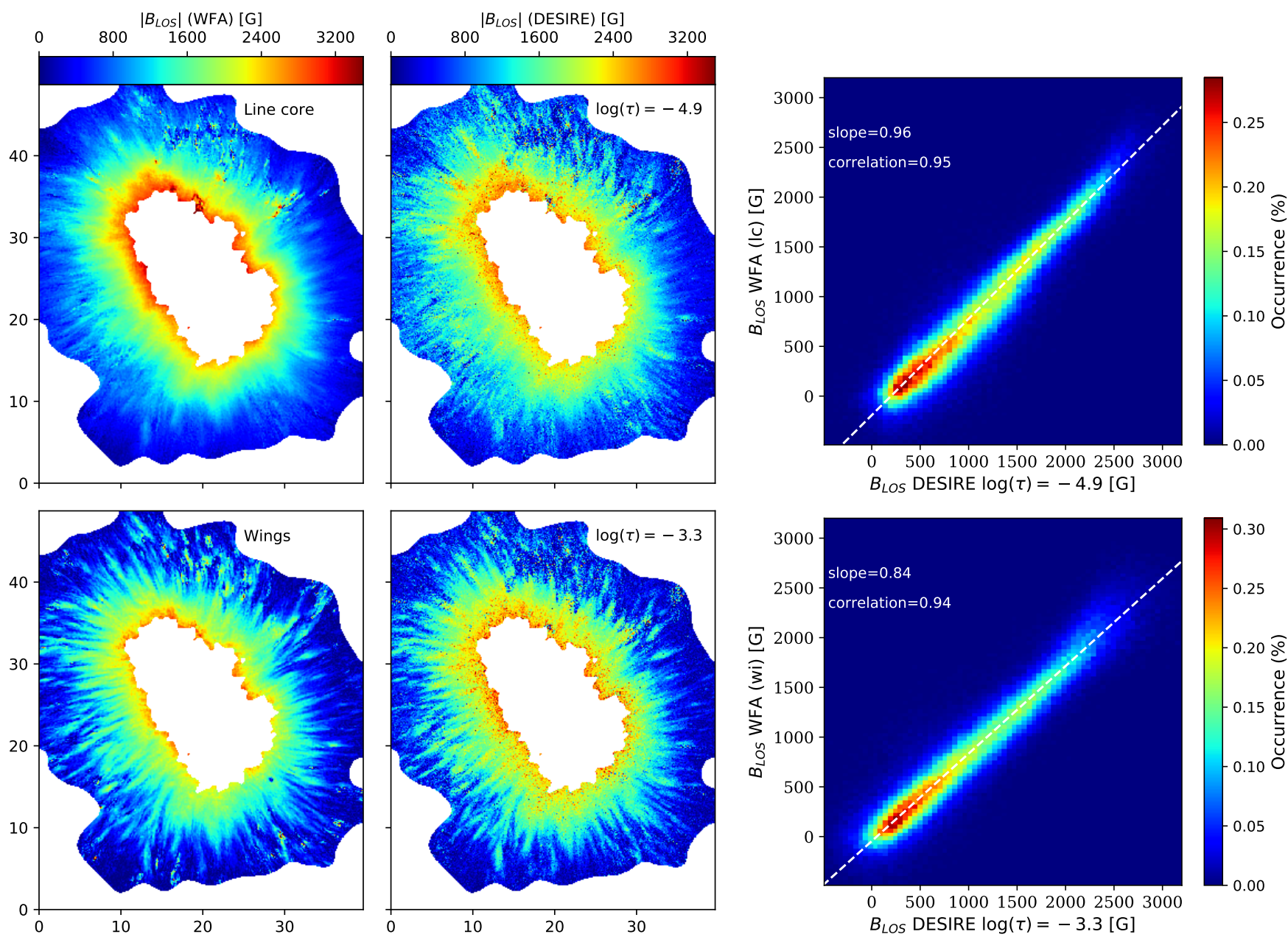

Fig. 8. Comparison between the $B_{\text {LOS }}$ values obtained in the sunspot penumbra (same time step as in Fig. 7) using the WFA and DeSIRe inversions. Left column: results from the WFA applied to the line core (top) and to the wings (bottom). Central column: inversion result at $\log (\tau)=-4.9($ top $)$ and $\log (\tau)=-3.3$ (bottom). Right column: density plots comparing the values from the two methods. We consider only penumbral pixels where $\chi^{2}<3$ is obtained with both methods. White dashed lines are regression lines.

can be seen in the upper maps, as expected for the chromospheric layers. The general appearance of the penumbra is reasonable, with the magnetic field decreasing with height and the field inclination becoming more vertical in the chromosphere at a fixed radial distance, as expected. The weakening of the field with height is not dramatic since the compared layers are not so distant from each other.

Also, we must bear in mind that the spectral line was observed in a relatively small wavelength range in order to get a sufficiently fast temporal cadence, which means that we have sampled only a small part of the wings and therefore, the photospheric information that can be retrieved from such a wavelength range is essentially limited to the upper photosphere.

\subsection{Inversions}

We compare the results obtained from the WFA in the sunspot (same FOV as displayed in Fig. 7) with inversions of the Stokes profiles carried out with the DeSIRe (Departure coefficient Stokes Inversion based on Response functions) code ${ }^{1}$. DeSIRe allows for the inversion of spectral lines that form under non-LTE conditions by combining the RH forward

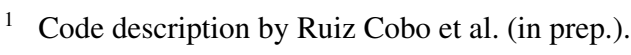

synthesis code (Uitenbroek 2001) and the SIR inversion code (Ruiz Cobo \& del Toro Iniesta 1992).

We invert the four Stokes parameters of the Ca II $8542 \AA$ line to infer the atmospheric properties within the formation region of the line. The inversion setup considers a simple onecomponent model atmosphere with typical values of the magnetic field strength $B$ and inclination $\gamma$, the LOS velocity $v_{\text {LOS }}$ and the temperature stratification between the photosphere and upper chromosphere (FALF model, Fontela et al. 1993). We use four cycles with five, six, seven, and nine equidistant nodes in temperature for each cycle; five nodes in micro-turbulence and LOS velocity; three nodes in the magnetic field strength, inclination and azimuth angles; and one node in macro-turbulent velocity.

Similarly to what Bellot Rubio et al. (2006) did for bisector velocities, we have determined the optical depth at which the inversion results are best comparable with the results from the WFA applied to the line core wavelengths, which have a maximum sensitivity in the range $\log (\tau)=[-4.5,-5.5]$. The wings of the line are sensitive to a range of optical depths $\log (\tau)=[0,-4]$ (Quintero Noda et al. 2016). We restrict the comparison to those pixels where the inversions perform relatively well (with $\chi^{2}<3$, which represent almost $65 \%$ of the pixels in the penumbra). Figure 8 displays such a comparison. In the line core (upper 


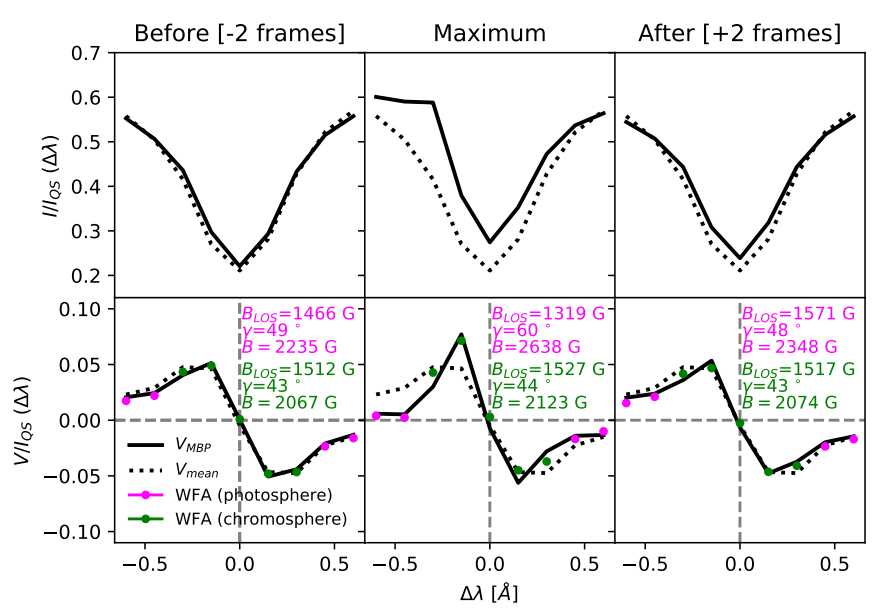

Fig. 9. Observed Stokes $I$ and $V$ profiles (solid lines) and temporally averaged profiles (dotted lines) at the MBP in PMJ 1 during three different temporal stages. The labels on each panel indicate the $B_{\mathrm{LOS}}, \gamma$ and $B$ values inferred from Eqs. (5) and (6), evaluated in the line wings (magenta) and the line core (green). The profiles resulting from the WFA fits are shown with magenta and green markers for the wings and the line core, respectively.

panels), the WFA has a maximum correlation with the inversions at $\log (\tau)=-4.9$. In the wings (lower panels), the maximum correlation is obtained at $\log (\tau)=-3.3$.

In spite of the considerably noisier maps obtained from the inversions, the solutions are highly consistent with the WFA results in both layers. The general appearance of the penumbra and most of the prominent penumbral structures can be reproduced with both methods with a large correlation between them. This is reflected in the density plots on the right column of the figure, which show a strong correlation between the WFA in the line core and the inversion results at $\log (\tau)=-4.9$ (low chromosphere), with a slope of 0.96 and a correlation coefficient of 0.95 . Likewise, there is a good correlation between the WFA in the wings and the inversion output at $\log (\tau)=-3.3$ (upper photosphere), with a correlation coefficient of 0.94 .

Therefore, the inversions provide a solution that is consistent with the WFA results. However, given that we have scanned the line at only a few wavelength points across a small wavelength range in order to maximize the temporal resolution of the data, we consider that the spectral information is not sufficient to perform inversions reliably. The WFA is a more robust method in this case, so from now on we use the WFA to investigate the magnetic properties of our PMJs.

\section{Results}

In this section, we describe the temporal evolution of the magnetic field inside and around PMJs, as inferred with the WFA in the upper photosphere and low chromosphere. We first concentrate on three different cases, and afterward we classify the evolution of all 36 PMJs based on how the field behaves during the maximum brightness stage with respect to its configuration during the frame before. Some statistics are also discussed.

\subsection{Case 1}

Figure 9 shows the Stokes $I$ and $V$ profiles emerging from the MBP of PMJ 1 at the times before, maximum, and after, as well as the result of applying the WFA to the line wings (magenta
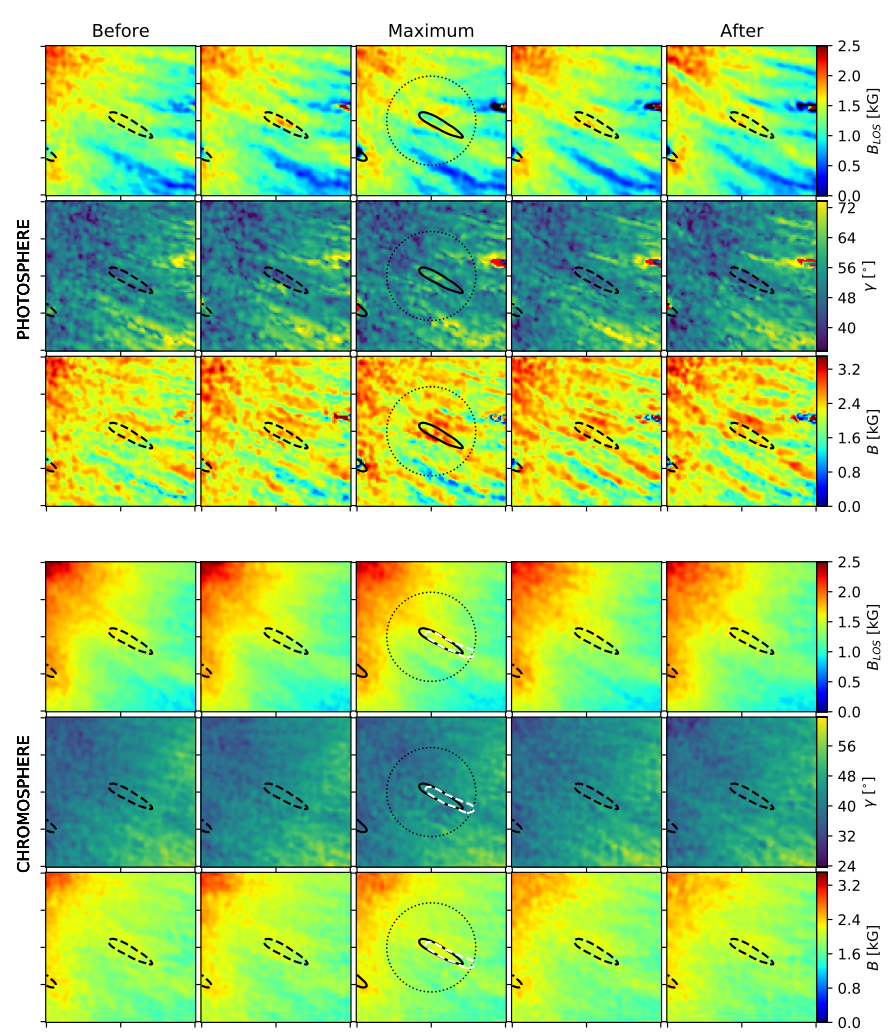

Fig. 10. Maps of $B_{\mathrm{LOS}}, \gamma$, and $B$ during the evolution of PMJ 1, computed by applying the WFA to the wings (first to third row) and to the line core (fourth to sixth row). The red patches in the inclination maps show regions where $\gamma>90^{\circ}$. The black contours enclose the PMJ area. Dotted circles on the maps at maximum define an arbitrarily chosen surrounding of the PMJ, centered on the MBP with a radius of $1.5^{\prime \prime}$. The columns show consecutive frames separated by $17 \mathrm{~s}$. The white contours enclose the CC region. The boxes cover a subfield of $5^{\prime \prime} \times 5^{\prime \prime}$.

markers) and to the line core (green markers). The shapes of the observed Stokes $V$ profiles can be nicely reproduced under the WFA, with $\chi^{2}<2$ in both wavelength ranges.

As indicated in the figure (magenta labels), there is a clear response of the photospheric field to the PMJ: the longitudinal field undergoes a reduction of nearly $150 \mathrm{G}$, which is accompanied by an increase in the inclination of around $10^{\circ}$, and by an increase in the total field strength of about $400 \mathrm{G}$ during the maximum. The configuration of the photospheric field almost recovers its initial state after the PMJ disappears.

The changes of the magnetic field in the lower chromosphere are substantially weaker than in the photosphere for the MBP (green labels), with $B_{\mathrm{LOS}}, \gamma$, and $B$ increasing by $15 \mathrm{G}, 1^{\circ}$, and $50 \mathrm{G}$ respectively from the stage before to the maximum. The magnetic field configuration at the stage after is remarkably similar to the stage before. The small magnetic field variations inferred for the low chromosphere are comparable to the uncertainties associated with the WFA, so they cannot be unambiguously related to the PMJ. What is clear is that the enhanced circular polarization signal observed at $\Delta \lambda=-0.15 \AA$ is mainly a consequence of the emission peak in the blue wing of the intensity profile, given that the shape of Stokes $V$ follows the shape of the derivative of Stokes $I$ scaled to a $B_{\mathrm{LOS}}$ on the order of 1.5 $\mathrm{kG}$ in the line core wavelengths.

In Fig. 10, we display maps of the magnetic field evolution in the upper photosphere and the low chromosphere during PMJ 1. The entire PMJ region (delimited by elongated black 


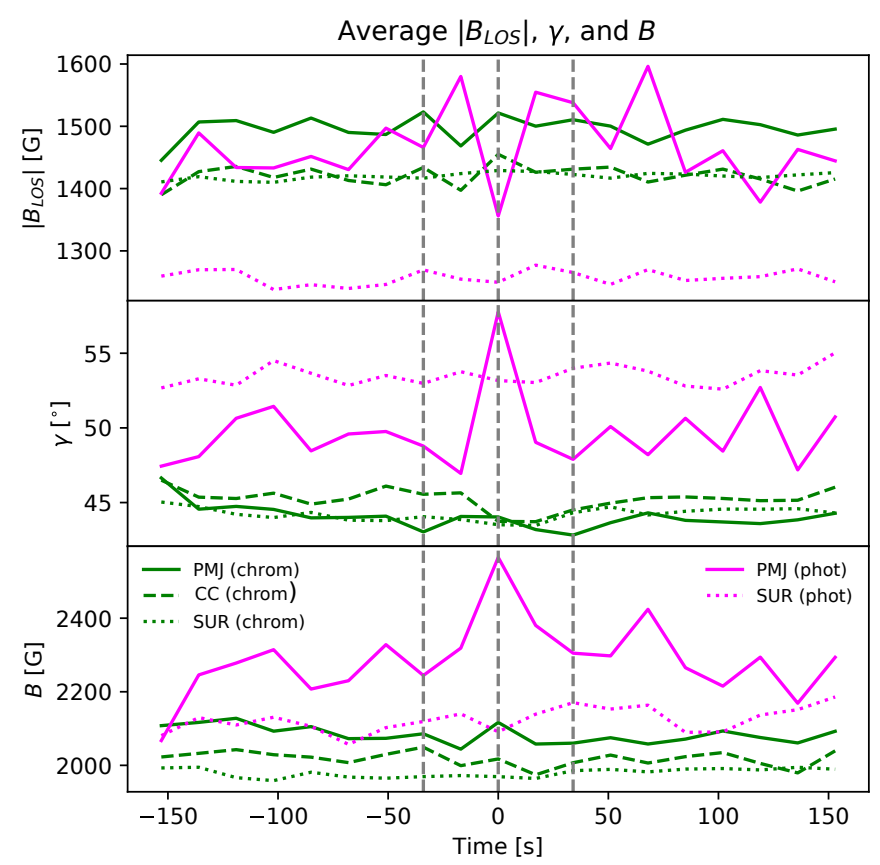

Fig. 11. Temporal evolution of $B_{\mathrm{LOS}}, \gamma$, and $B$ averaged within PMJ 1 (solid lines), the $\mathrm{CC}$ region (dashed lines), and in the surroundings along the dotted circles drawn in Fig. 10 (dotted lines) in the lower chromosphere (green) and upper photosphere (magenta). The vertical dashed lines mark the stages before, maximum, and after.

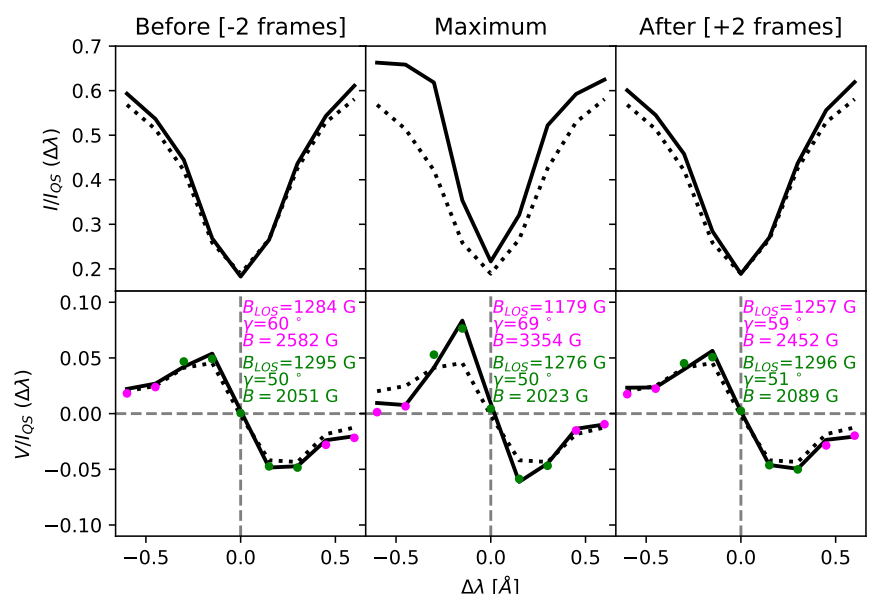

Fig. 12. Observed Stokes $I$ and $V$ profiles and results of the WFA at the MBP in PMJ 2 (same format as Fig. 9).

contours) displays magnetic field changes that are qualitatively similar to those observed at the MBP during the three stages of interest, namely, the photospheric field becomes stronger and more inclined during the maximum brightness stage, when it displays the largest changes. This can be clearly observed in Fig. 11, where the temporal evolution of the average photospheric magnetic field inside the PMJ region is shown with magenta solid lines.

With an average magnetic field inclination of $\sim 50^{\circ}$ in the photosphere and a height difference of $\sim 500 \mathrm{~km}$ between the photosphere and the low chromosphere, the projection of the CCs would be about 15 pixels, in agreement with the observed shifts. The plots of Fig. 11 reveal that there are not clear changes occurring in the chromospheric field either along the LOS or along the magnetic field lines in this case. The field inside the
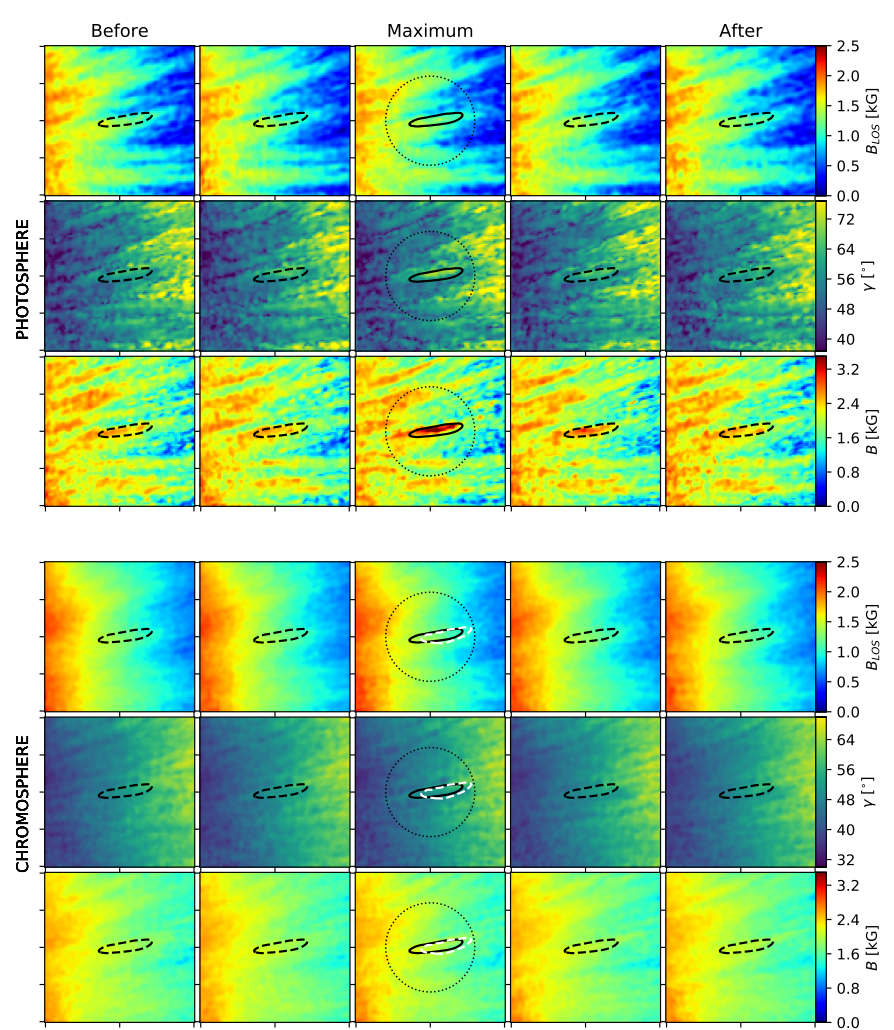

Fig. 13. Maps of $B_{\mathrm{LOS}}, \gamma$, and $B$ during the different stages of evolution of PMJ 2 (same format as Fig. 10).

PMJ and the CC regions show a rather steady evolution (green solid and dashed lines, respectively).

To investigate how the magnetic field behaves in the surroundings during PMJ 1, we averaged the magnetic field parameters along the circles displayed in Fig. 10. The dotted lines in Fig. 11 show the temporal evolution of the field which, as expected, does not undergo any evident change that can be clearly related to the PMJ occurrence, neither in the photosphere nor in the chromosphere. There are very small fluctuations during the $300 \mathrm{~s}$ temporal range of the plots, which might be due to seeing or instrumental effects, and even minor changes occurring in the penumbral field. In the photosphere, the fluctuations of the field inside the PMJ region are slightly larger than those in the surroundings. Nonetheless, the changes observed at maximum in the PMJ region clearly stand out, which means that such changes are not systematic variations.

\subsection{Case 2}

Figures 12 and 13 show the temporal evolution of PMJ 2, whose brightening is observed during three consecutive frames in the limb-side penumbra (green marker in Fig. 1). It occurs near the end of an inner penumbral filament. As shown in Fig. 12 for the MBP, the emergent Stokes I profiles are remarkably similar during the stages before and after. In contrast, the entire profile displays an increase in the intensity during the maximum stage, and such brightening is notably larger in the blue wing wavelengths, similar to the MBP of PMJ 1.

The results of the WFA indicate that the photospheric longitudinal field decreases almost $100 \mathrm{G}$ from the frame before to the frame maximum, whilst the field inclination increases by nearly $10^{\circ}$ and the total field strength increases more than $700 \mathrm{G}$ 


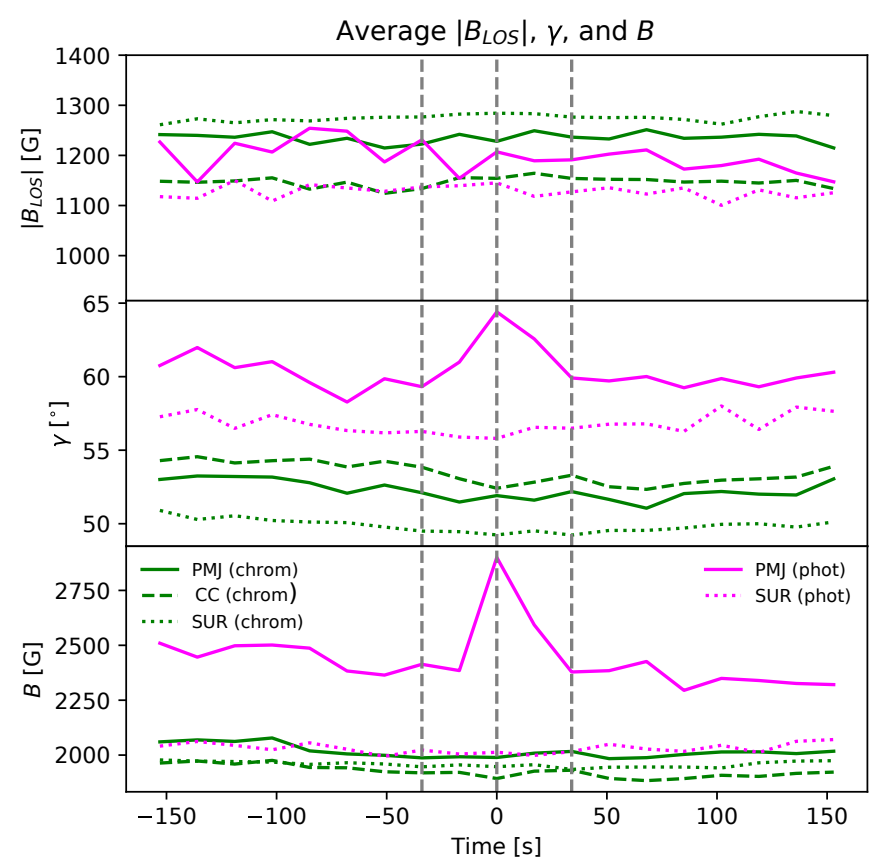

Fig. 14. Temporal evolution of $B_{\mathrm{LOS}}, \gamma$, and $B$ inside and in the surroundings of PMJ 2 (same format as Fig. 11).

between the two stages. Afterward, the photospheric magnetic configuration in the stage after returns to almost the same state as in the stage before. Also, similarly to PMJ 1, there are not substantial changes of the chromospheric field.

However, Figs. 13 and 14 show that, unlike PMJ 1, the average photospheric $B_{\mathrm{LOS}}$ inside PMJ 2 does not display considerable changes with time. In this case, there is only a clear increase in the average field inclination and strength in the photosphere, while there are not clear signatures in the evolution of the chromospheric field that can be associated with PMJ 2, either along the LOS or along the same field lines. Figure 13 also shows a more inclined field inside the PMJ region than in the immediate surroundings in all the frames, a result that is consistent with the observation of an enhanced TLP signal inside PMJ 2 since the stage before (Fig. 6).

Despite the differences in $B_{\mathrm{LOS}}$, the evolution of the photospheric field is similar for PMJs 1 and 2: there is a clear increase in $\gamma$ and $B$ in both cases. Slight differences in the behavior of the magnetic field are expected for PMJs that occur on different penumbral structures, which is the case of PMJs 1 and 2 as indicated by their different photospheric magnetic configuration.

\subsection{Case 3}

PMJ 3 was observed during four consecutive frames nearly perpendicular to the symmetry line of the sunspot (cyan marker in Fig. 1), above the interface between spines and intra-spines. Figures 15 and 16 show the evolution of the polarization signals in the MBP, located in the central part of the brightening region, and in a pixel located near the border of the PMJ, respectively. The MBP shows intensity enhancements in the entire profile at maximum, with both wings displaying emission, but a larger increase is observed in the blue wing. As a consequence of the strong emissions in the wings, the Stokes $V$ profile displays two extra lobes at maximum. At the stage after, the intensity profile displays a more regular shape with no wing emission, similar to the profile observed in the frame before. However, the line core

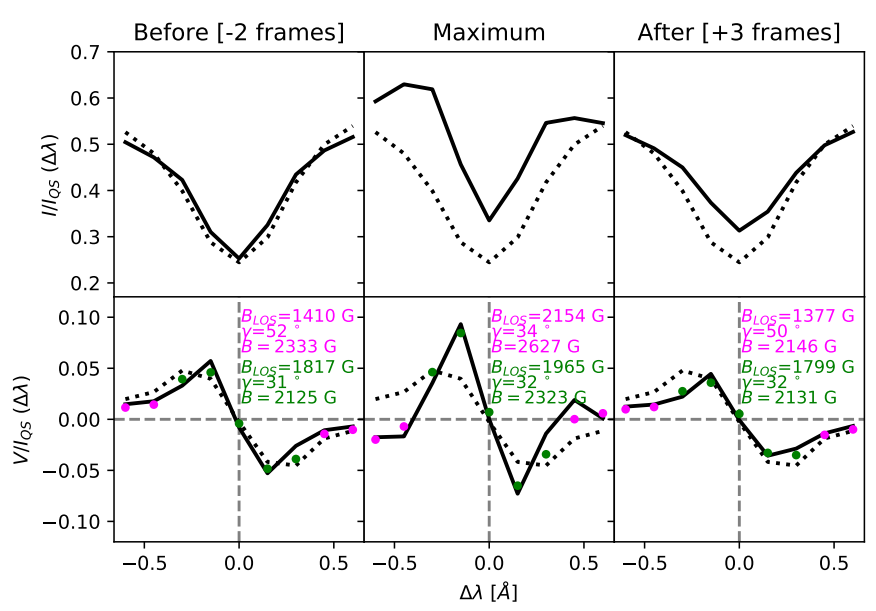

Fig. 15. Observed Stokes $I$ and $V$ profiles at the MBP in PMJ 3 and results of the WFA (same format as Fig. 9).

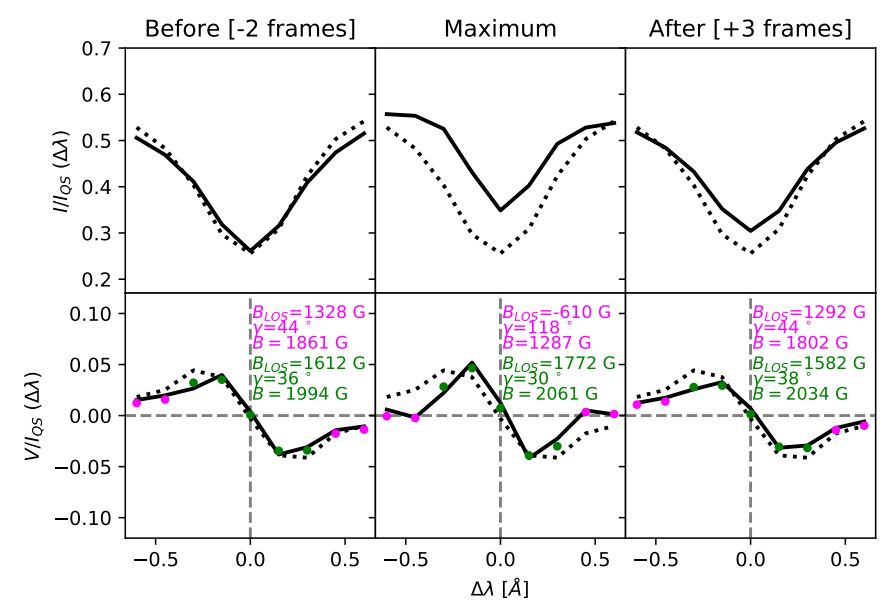

Fig. 16. Observed Stokes $I$ and $V$ profiles in a pixel near the border of PMJ 3 and results of the WFA (same format as Fig. 9).

remains notably brighter than before. For this pixel, the WFA infers an increase in the photospheric magnetic field strength of nearly $300 \mathrm{G}$ and a strong decrease in the inclination of $18^{\circ}$ that results in an enhancement of $B_{\mathrm{LOS}}$ by about $700 \mathrm{G}$ between the stages before and maximum.

The profiles at the edge of the PMJs (Fig. 16) also show intensity enhancements in the entire wavelength range at maximum, but unlike the MBP, in this case the wings are not in emission (i.e., there is not a change of sign in the derivative of $I$ ). Nonetheless, the Stokes $V$ profile displays reduced signals in the wings with opposite signs to the profiles from the stages before and after. This is interpreted as a change of polarity in the photospheric magnetic field by the WFA, which also infers a decrease in the magnetic field strength of more than $500 \mathrm{G}$ and an increase in the inclination in the photosphere.

The different behaviors observed in PMJ 3 at maximum can also be noticed in the maps of Fig. 17: in the upper photosphere, the magnetic field becomes stronger and more vertical in the central part of the PMJ region (similar to the MBP), while it turns weaker and more inclined in the inner borders. Furthermore, the photospheric field seems to change polarity in some pixels at the edges of the PMJ region. It is difficult to judge if such polarity changes are real given that the Stokes $V$ wing signals are very 

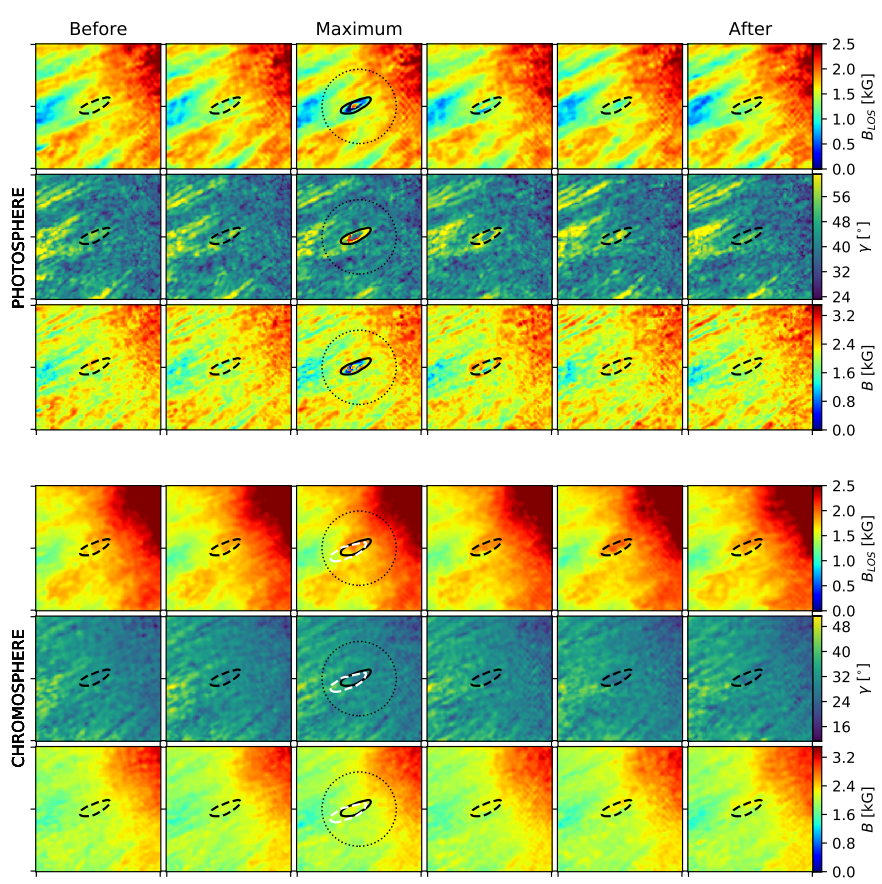

Fig. 17. Maps of $B_{\mathrm{LOS}}, \gamma$, and $B$ during the different stages of the evolution of PMJ 3 (same format as Fig. 10).

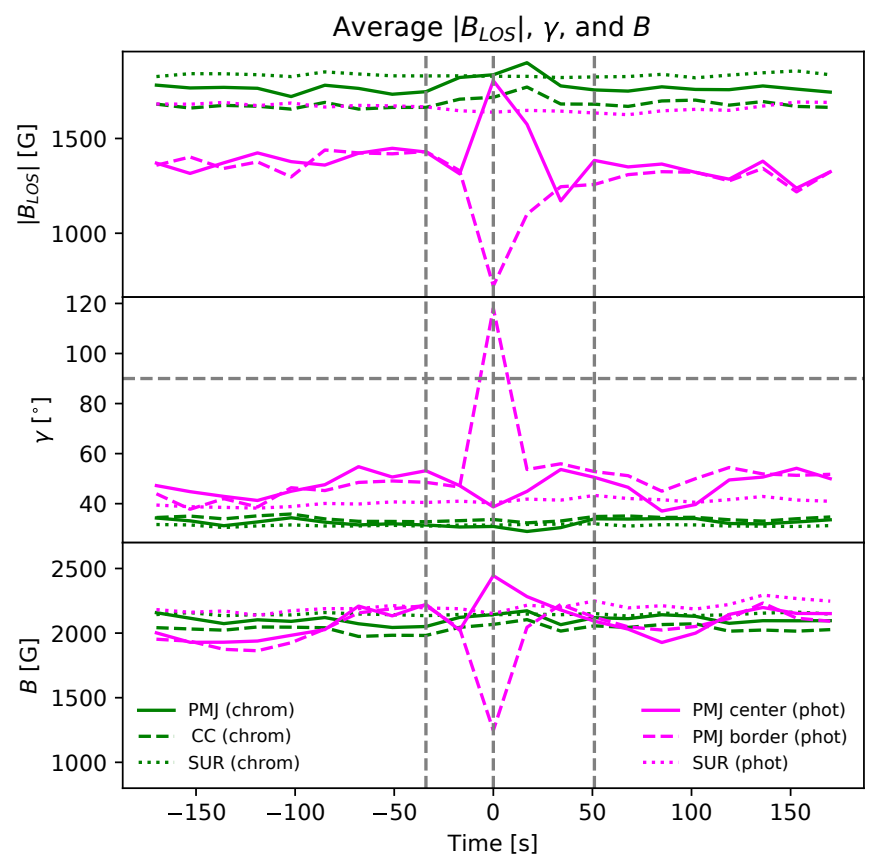

Fig. 18. Temporal evolution of $B_{\mathrm{LOS}}, \gamma$, and $B$ in different regions during PMJ 3: for the photosphere (magenta lines), in the central part of the PMJ (solid), edges of the PMJ region (dashed), and surroundings (dotted); for the chromosphere (green), in the PMJ (solid), the CC region (dashed), and the surroundings (dotted).

small in those pixels and even fall within the level of the noise, similarly to the profiles in Fig. 16.

The magenta solid lines in Fig. 18 show the temporal evolution of the average photospheric $B_{\mathrm{LOS}}, \gamma$, and $B$ in the central part of the PMJ region, which contains the MBP. The evolution of the average field in the borders of the PMJ region is shown with dashed lines. The differences in the behavior of the
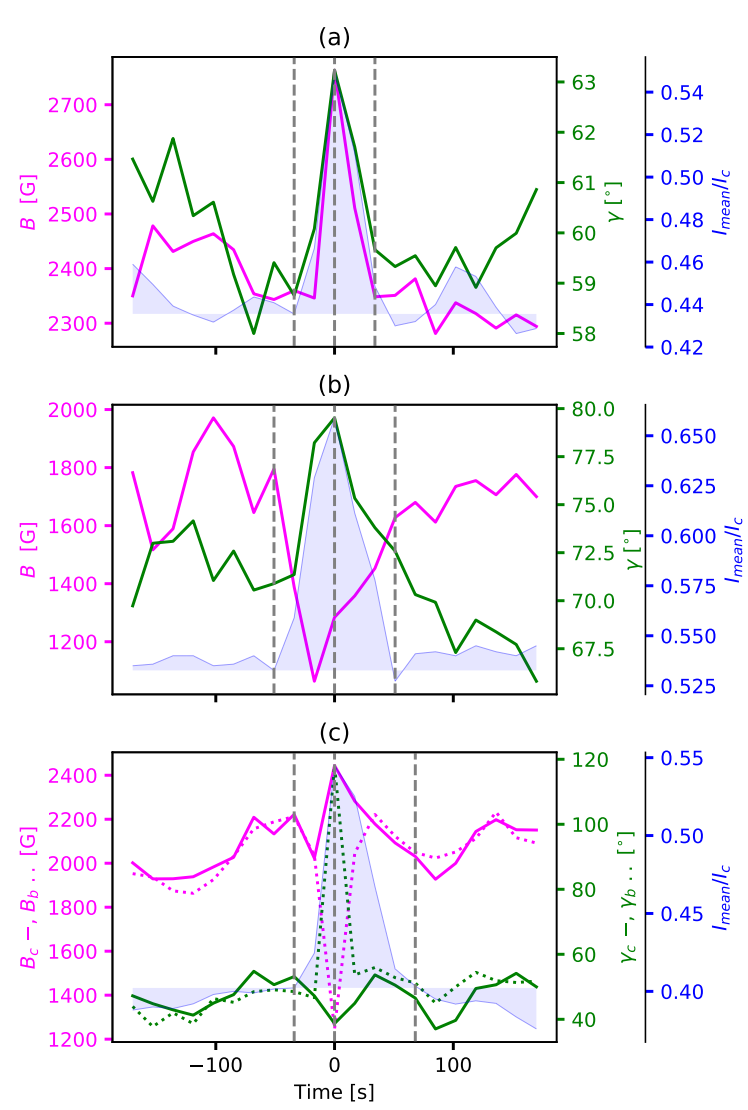

Fig. 19. Three different types of temporal evolution of $\gamma$ (green) and $B$ (magenta) observed among the 36 PMJs in the upper photosphere. Values are averaged inside the PMJ area for the cases shown in $(a)$ and $(b)$, but the averaging is done separately in the central region (solid lines) and in the borders of the brightening region (dotted lines) for case $(c)$. Blue shades show the light curves in the PMJ calculated by averaging the intensity in the blue wing of the line. The vertical dashed lines in each panel indicate the three stages of interest, with the maximum brightness stage placed at the origin.

photospheric field become more evident in these plots. However, the two regions do not display big differences before and after the PMJ occurrence. In the low chromosphere (green), the whole PMJ region displays an increase in $B_{\mathrm{LOS}}$ and $B$ (solid lines). A similar response is observed in the $\mathrm{CC}$ region (dashed lines in Fig. 18). Although these changes are still weaker than in the upper photosphere, they appear to be clearly associated with the PMJ brightening. As in PMJs 1 and 2, the surroundings of PMJ 3 do not display changes of magnetic configuration during the PMJ occurrence (see the dotted lines in Fig. 18).

\subsection{Magnetic field evolution in the photosphere}

All 36 PMJs displayed considerable changes of the photospheric magnetic field configuration. Taking into account the evolution of the average $\gamma$ and $B$ inside the brightening regions, we identify three types of PMJs as shown in Fig. 19.

Most of the PMJs (25 out of 36 PMJs, or 69\%) show a magnetic field evolution of type (a) similar to those of PMJs 1 and 2 , that is, they undergo a transient increase in the field inclination and the field strength during the maximum brightness phase. PMJs of type (a) are indicated with red markers in Fig. 2. These PMJs tend to appear slightly clustered in the limb-side 

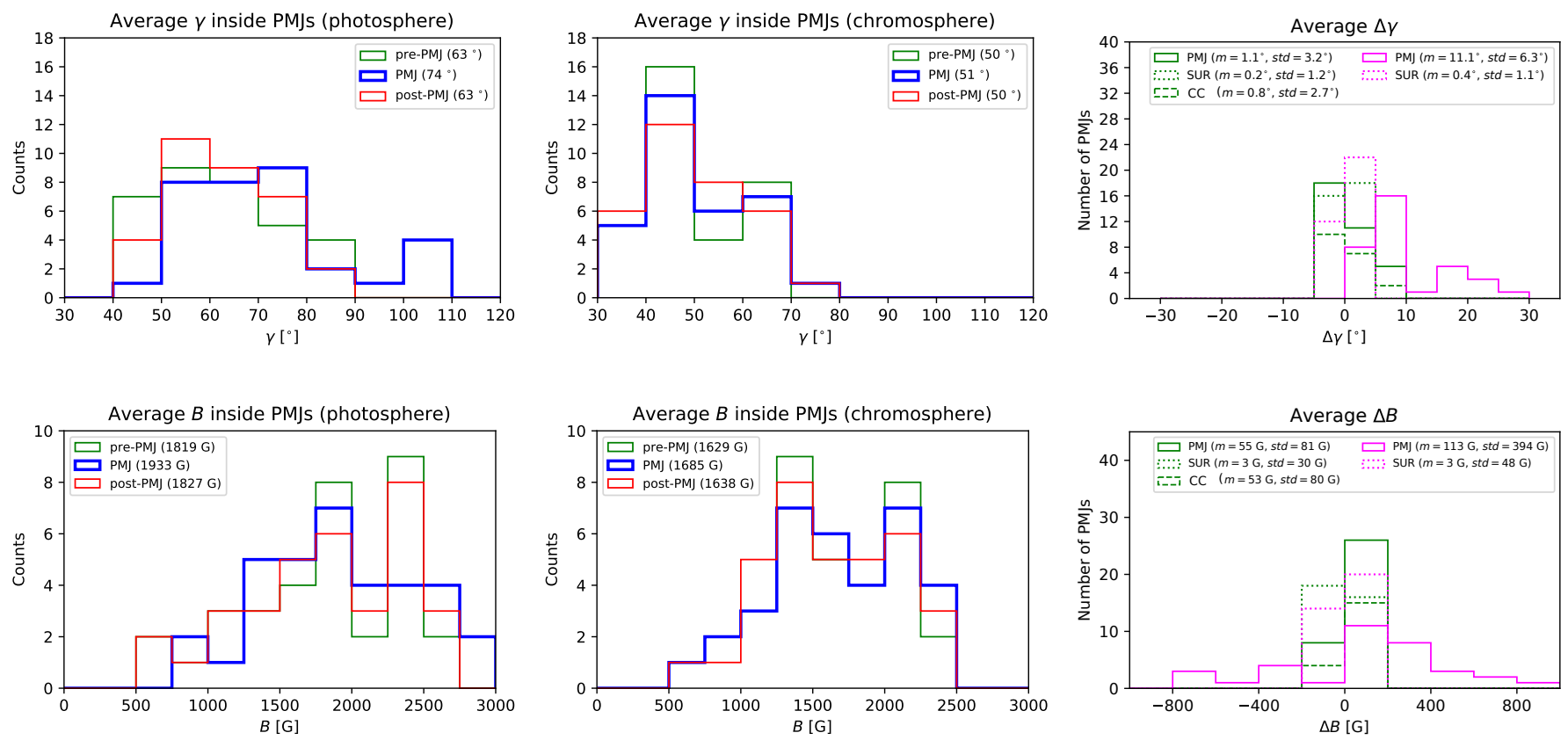

Fig. 20. Magnetic field configuration of 34 PMJs in the upper photosphere (left) and low chromosphere (center). The histograms show the average values of $\gamma($ top $)$, and $B$ (bottom) inside the PMJ regions during three different stages: the pre-PMJ phase (green), which corresponds to the average field during 2 min before the PMJ, the maximum brightness (blue), and the post-PMJ phase (red), which is the average field during 2 min after the PMJ. The legends give the mean values of each distribution. Right: distribution of magnetic field changes between the pre-PMJ phase and the maximum brightness in the photosphere (magenta) and in the chromosphere (green) inside the PMJ regions (solid) and in the surroundings (dotted). The green dashed distributions show the chromospheric field changes occurring inside the CC regions for the 19 PMJs marked with circles in Fig. 2. The legends give the mean $m$ and the standard deviation $s t d$ of each distribution.

penumbra, but they can be seen all over the penumbra and in two cases even right at the outer penumbral boundary.

Small differences are detected among the PMJs of type (a), mainly due to the behavior of $B_{\mathrm{LOS}}$. Specifically, in 16 of them the longitudinal field decreases as a consequence of the increase in the inclination, similar to PMJ 1 . In four cases, $B_{\mathrm{LOS}}$ does not display clear changes in spite of the increase in $\gamma$ and $B$, as happens in PMJ 2. In five PMJs of type (a), observed near the outer limb-side penumbral boundary where the magnetic field is weak and largely horizontal, the WFA infers a change of polarity during the maximum brightness stage. However, in all cases the Stokes $V$ profiles show reduced signals in the wings, down to the noise level, which makes these polarity changes uncertain.

Another type of evolution is shown in Fig. 19b and was observed in nine PMJs (25\% of the cases; blue markers in Fig. 2 ). In this type of evolution, the photospheric magnetic field vector also becomes more inclined, but the field strength shows an overall decrease during the brightening phase.

Finally, there are two PMJs whose brightening regions show mixed types of magnetic field evolution (5\% of the sample; green markers in Fig. 2). One of them is PMJ 3. In these cases, the photospheric field becomes stronger and slightly more vertical in the central part of the brightening, but weaker and more inclined near the borders, as shown in Fig. 19c. Interestingly, the WFA infers changes of magnetic polarity in some pixels near the border of the brightening region in both PMJs, but again this result requires further confirmation due to the very small Stokes $V$ signals observed in the line wings.

The left panels of Fig. 20 show distributions of the average photospheric magnetic field in 34 PMJs (we exclude the two PMJs of type (c) to avoid mixing different magnetic configurations) during three stages of interest: the pre-PMJ phase, the maximum brightness, and the post-PMJ phase. The pre- and
post-PMJ phases correspond to the average magnetic field over nearly $2 \mathrm{~min}$ (seven consecutive frames) before the start and after the end of the PMJs, respectively. The two-minute averaging is made to smear out the largest fluctuations.

In all three phases, the distributions cover a wide range of values of $\gamma$ and $B$, due to the fact that the PMJs occur at different radial distances within the penumbra. However, the distributions for the maximum brightness stage (blue) stand out clearly from the pre- and post-PMJ distributions (green and red, respectively), since they contain more inclined and stronger fields. Specifically, the mean values of $\gamma$ and $B$ at the time of maximum brightness are larger that those observed during the pre- and post-PMJ phases by $\sim 10^{\circ}$ and $\sim 100 \mathrm{G}$, respectively. In contrast, the distributions for the pre- and post-PMJ phases are quite alike and have very similar mean values, which means that no strong permanent changes in the local photospheric field were produced by the occurrence of the PMJs.

The right panels of Fig. 20 show distributions of the photospheric magnetic field changes occurring in the surroundings of the PMJs (magenta dotted lines), defined by those pixels that lie along circumferences of $1.5^{\prime \prime}$ radius centered at the MBPs. The changes in the surroundings are always much smaller than the ones observed in the PMJ regions, by a factor of at least 10 . This shows that PMJs are highly localized events that do not leave imprints on the adjacent medium.

\subsection{Magnetic field evolution in the chromosphere}

The magnetic properties of the short-lived PMJs exhibit weaker changes in the chromosphere than in the photosphere (see green solid distributions in the right panels of Fig. 20). In most of the cases, it is difficult to judge if those changes are caused by the PMJs because either they are of the same order of magnitude 


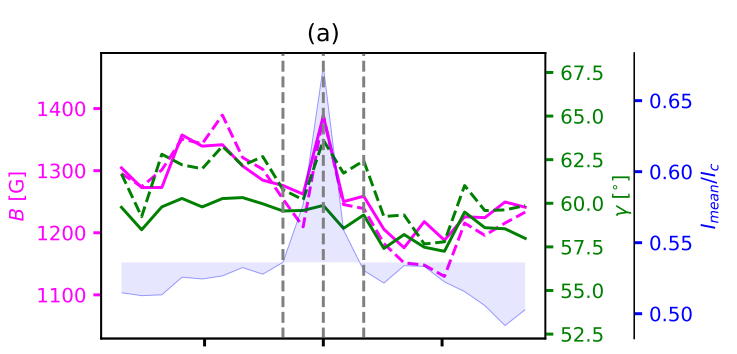

(b)

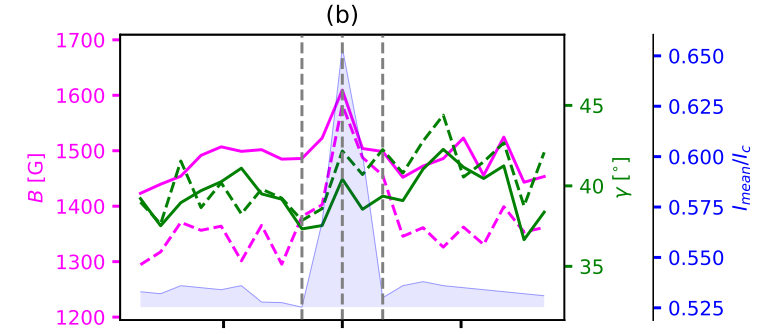

(c)

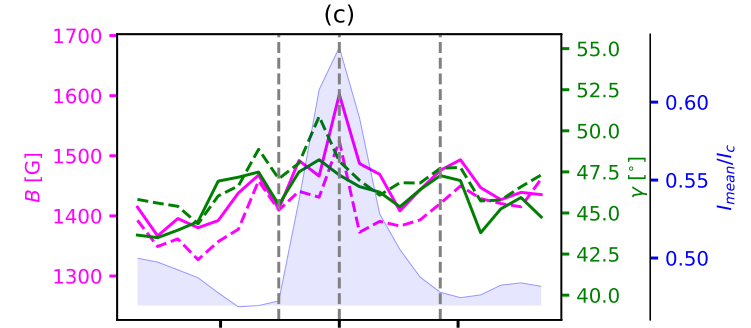

(d)

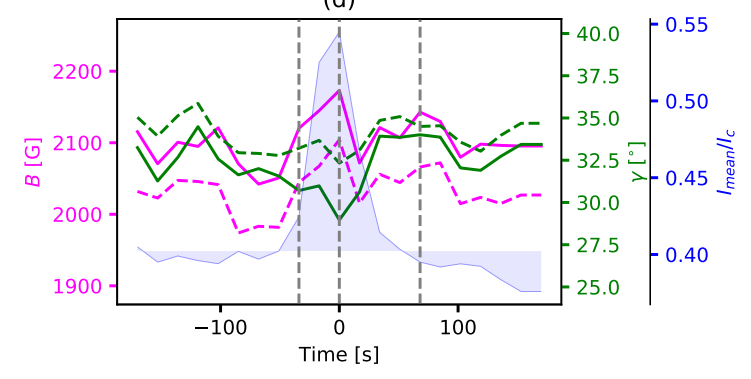

Fig. 21. Temporal evolution of $\gamma$ (green) and $B$ (magenta) in the chromosphere as inferred from the WFA for the individual PMJs marked with crossed symbols in Fig. 2. The solid lines show the average values inside the PMJ regions and the dashed lines show averages inside the CC regions (same format as Fig. 19).

as the errors associated with the WFA or they cannot be distinguished from other small-scale fluctuations that might be intrinsic to the penumbral field.

However, Fig. 21 shows four PMJs (crossed symbols in Fig. 2) displaying some degree of chromospheric response to the observed brightenings inside both the PMJ (solid lines) and the $\mathrm{CC}$ regions (dashed lined). These PMJs occur with a slight but clean increase in the total field strength in the chromosphere at maximum, while there is not a clear trend in the evolution of the magnetic field inclination. The observed changes have similar amplitudes inside the PMJ and within the $\mathrm{CC}$ regions, except in the PMJ of Fig. 21b, where the increase in the magnetic field strength is about twice as larger inside the $\mathrm{CC}$ region as that inside the PMJ.

The central panels of Fig. 20 show that the distributions of the average $\gamma$ and $B$ in the chromosphere inside the PMJ regions are remarkably similar during the pre-PMJ, maximum, and postPMJ phases. They have mean values of $\sim 50^{\circ}$ and $\sim 1600 \mathrm{G}$ respectively. However, the average value of $B$ for the distribu- tions at maximum is slightly larger than for the pre- and postPMJ phases by about $50 \mathrm{G}$.

The right panels of Fig. 20 show distributions of the chromospheric magnetic field changes occurring inside the PMJ regions (solid green lines). There is about the same number of PMJs becoming slightly more vertical and more inclined than the prePMJ phase, with a mean $\Delta \gamma \sim 1^{\circ}$. This value is well below the mean $\Delta \gamma$ of the photospheric field (magenta solid distribution). In contrast, the chromospheric field tends to be stronger during the maximum brightness stage in most PMJ regions. The distribution has a mean $\Delta B$ and a standard deviation of 55 and $81 \mathrm{G}$, respectively. However, these values are about two and five times smaller than those in the upper photosphere.

Unlike the four PMJs of Fig. 21, the majority of the identified $\mathrm{CC}$ regions did not show clear changes of the chromospheric field configuration, similar to the PMJ regions. The distribution of their changes are shown with green dashed lines in the right panels of Fig. 20 and display remarkably similar trends and mean values as those of the green solid distributions.

The chromospheric field changes occurring in the surroundings of the PMJs (dotted green distributions) are yet smaller than the chromospheric changes inside the PMJ and CC regions. This means that even if very small, the changes of the chromospheric field observed during the PMJs along the LOS and along the field lines are likely not a result of systematic errors.

\section{Summary}

We have presented a detailed analysis of the magnetic field evolution during the lifetime of 36 short-lived PMJs. Our investigation focused on the configuration of the magnetic field in the upper photosphere and low chromosphere based on the WFA applied separately to the wings and core of the Ca II $8542 \AA$ line. The main results of this analysis can be summarized as follows:

Firstly, the PMJs showed brightness enhancements in at least one of the wings and frequently also in the line core, but the enhancement was always larger in the blue wing than in the red wing (above $10 \%$ and up to $63 \%$ of the temporally averaged intensity over the full time series).

Secondly, 19 PMJs displayed line-core intensity enhancements exceeding $10 \%$ of their temporally averaged values in regions that are shifted outward by $10-25$ pixels in the radial direction from the PMJ regions, which correspond to distances of $\sim 400-1000 \mathrm{~km}$. They roughly match the expected projections on the POS of the CCs of field lines with inclinations of $40-65^{\circ}$ in the upper photosphere. In many other cases, the line core remained practically unchanged in the PMJ region and surroundings so that we could not detect the CCs. It could be that the field lines are largely horizontal and do not reach the chromosphere within our FOV.

In the $\mathrm{CC}$ regions, the intensity profiles commonly showed slightly brighter cores even after the PMJ disappeared, lasting at least $85 \mathrm{~s}$ longer (five frames).

Thirdly, all 36 PMJs displayed enhanced polarization signals in Stokes $Q, U$, and $V$ at maximum. In particular, the Stokes $V$ profiles presented enhanced signals within the line core region $(|\Delta \lambda| \leq 0.30 \AA)$, but in most cases the Stokes $V$ signals in the wings $(0.45 \AA \leq|\Delta \lambda| \leq 0.60 \AA)$ became weaker at maximum. In some cases, the PMJs display extra lobes in the wings due to emission or possible changes of polarity of the photospheric field. This resulted in different types of changes of the TCP during the maximum stage, with most of the PMJ regions showing a slight decrease or no clear changes in TCP 
and others displaying an enhanced TCP with respect to the stages before and after. In contrast, all PMJs exhibited enhanced TLP at maximum.

Positive NCP values were commonly observed inside the PMJs. They can be understood as a result of the asymmetries of the intensity profiles, which display larger emission peaks in the blue wing than in the red wing. Such asymmetries affect the shapes of the Stokes $V$ profiles because they follow the shapes of the partial derivatives of Stokes $I$ with wavelength. Hence, since the sunspot has positive polarity and the positive blue lobe amplitudes and areas were predominantly larger than those of the negative red lobes, the NCP values turned out to be positive during the PMJs. They could also be partly the result of $B$ and $v_{\text {LOS }}$ gradients, as it is the case of photospheric lines. Inversions are needed to confirm or disprove this mechanism.

The WFA applied to the wing wavelengths reveals clear changes of the photospheric magnetic field configuration inside the PMJ regions. These changes were generally strongest at the maximum brightness stage. We identified three different types of evolution of the photospheric magnetic field, but in most cases the field inclination and the field strength increase at maximum. In contrast, the WFA applied to the line core wavelengths suggests very small changes of the chromospheric field. Nonetheless, there is a trend of having slightly stronger chromospheric fields inside the $\mathrm{PMJ}$ and $\mathrm{CC}$ regions at maximum compared to their pre- and post-PMJ phases. Therefore, one could say that the enhanced polarization signals of the line core wavelengths are mostly induced by the observed intensity enhancements rather than by strong changes in the chromospheric magnetic field.

The chromospheric magnetic field changes are remarkably similar when we look along the LOS or along the same field lines. This is because the projection effects were relatively small in most of the identified CC regions.

Only four PMJs displayed clear changes of the chromospheric field, which had a neat correlation with the brightness enhancement of the PMJs. In these cases, the field strength experienced an increase at maximum. However, such changes were much weaker than in the photosphere.

Finally, the changes of the photospheric and chromospheric magnetic field in the surroundings of the PMJs were negligible compared to the changes inside the PMJ regions, which rules out the possibility that the latter are caused by noise or intrinsic fluctuations in the penumbra.

These results show that the PMJ brightenings are indeed accompanied by a perturbation in the magnetic field. Such perturbation must originate closer to the height where the wings of the Ca II $8542 \AA$ line are formed, that is, the upper photosphere. These magnetic perturbations likely propagate but rapidly decrease toward the immediate surroundings of the PMJs, which show negligible changes beyond the brightening region. They likely also propagate upward to chromospheric heights where they leave weaker imprints. However, since the only chromospheric change detected seems to be an increase in the magnetic field strength, it is possible that only the compressive phase of the perturbation reaches the chromosphere.

\section{Discussion and conclusions}

This work provides the first analysis of the temporal evolution of the magnetic field vector in short-lived PMJs using spectropolarimetric observations with very high temporal resolution. In this section, we discuss our results and revisit some important aspects of PMJs reported in previous works.

\subsection{Location}

Most of the false detections in our dataset were caused mainly by distorted profiles due to the strong inverse Evershed flow or strongly red-shifted profiles repeatedly observed along superpenumbral filaments and appearing as elongated bright structures in the blue-wing image sequences as well as in the running difference images. Therefore, after a careful examination of nearly two hundred pre-selected events, we finally identified only 36 events as short-lived PMJs.

Similar to the findings of Esteban Pozuelo et al. (2019) for PMJs of longer duration, our short-lived PMJs occurred above regions that are expected to harbor strong horizontal gradients of the magnetic field inclination in the photosphere. This supports the scenario of magnetic reconnection taking place in the lower photosphere as a possible driver of PMJs (e.g., Tiwari et al. 2016).

\subsection{Lifetimes}

Reardon et al. (2013) find the presence of a precursor phase observed up to $1 \mathrm{~min}$ before the rapid impulsive brightening of some PMJs. This could be an indicator of disturbances in density or temperature occurring prior to a reconnection event, such as those due to bow-shocks. In our data, we have also observed a large fraction of PMJs displaying a discernible precursor phase. However, we have discarded such events given that their lifetimes were longer than the upper limit of two minutes chosen for this study.

The lifetime distribution of the 36 PMJs peaks at $68 \mathrm{~s}$ and has a mean value of $71 \mathrm{~s}$, which is lower than the $90 \mathrm{~s}$ mean lifetime found by Drews \& Rouppe van der Voort (2017). However, the number of events in our sample and the upper cutoff limit of 2 min for the durations are both much smaller than those considered by those authors. Hence, our samples are not comparable and differences in the lifetime distributions can be expected.

\subsection{Magnetic field evolution}

The shortest-lived PMJs show enhanced polarization signals in the Ca II $8542 \AA$ profiles with respect to their surroundings like long duration PMJs (Esteban Pozuelo et al. 2019). However, we found that the polarization signals do change with time inside the PMJ regions, displaying the largest amplitudes during the maximum brightness stage. According to the WFA, the short-lived PMJs produce different types of changes in the photospheric magnetic field, which are generally strongest at the maximum brightness stage. The different types of evolution displayed by the photospheric magnetic field do not seem to be related to the position of the PMJs within the penumbra. Thus, it is possible that not all the short-lived PMJs have the same nature. However, due to our temporal resolution of $17 \mathrm{~s}$, we may have captured different phases of the same type of perturbation, which we misinterpreted as different types of evolution of the magnetic field.

The changes identified in the chromospheric magnetic field, on the order of $100 \mathrm{G}$, are in agreement with the findings of Buehler et al. (2019), who observe an increase of nearly $100 \mathrm{G}$ in the LOS magnetic field associated with two PMJs when compared to the average field during the stages before and after. These authors also applied the WFA to Ca II $8542 \AA$, but using a wider spectral range $( \pm 765 \mathrm{m \AA})$. They also notice that the magnetic perturbation seemed to propagate on the POS with similar projected velocities as the PMJ brightenings move on such a plane. 


\subsection{Origin}

According to our results, it is possible that the observed changes of the magnetic field are caused by perturbation fronts that have a photospheric origin. This is supported by the fact that they are larger in the upper photosphere than in the chromosphere. Furthermore, the changes of the orientation and strength of the field suggest that the perturbations have a compressive nature, and therefore they likely propagate at oblique directions with respect to the magnetic field. Such upwardly propagating fronts would likely involve a compressive leading phase followed by a rarefaction phase which spreads out with time, similarly to a shock wave. Since we only observed a strengthening of the magnetic field in the chromosphere, it is possible that the rarefaction phase fades out as the front propagates upward in the atmosphere.

The magnetic field changes we have detected in PMJs cannot be caused by transverse waves propagating along the magnetic field since in that case we would observe only a change of orientation but not of the strength of the field. Therefore, we interpret the observed magnetic field variations as due to perturbations propagating across or obliquely to the field, such as compressional Alfvén waves or MHD fast-mode wavefronts, which likely steepen quickly as they travel upward in the photosphere, thus leading to the formation of a shock front similar to what occurs during umbral flashes (Grant et al. 2018). Buehler et al. (2019) also suggested that the magnetic perturbations observed in the chromosphere are likely produced by Alfvén waves.

\subsection{Temperature}

A temperature increase along the magnetic field lines that connect the upper photosphere with the low chromosphere would explain the intensity changes observed in different wavelengths and in different positions on the POS, and this is in agreement with the magnetic reconnection scenario. In particular, it is well known that the Ca II $8542 \AA$ line core can be a reasonable proxy for the temperature in the chromosphere (Cauzzi et al. 2009). Therefore, the slightly brighter line cores observed during the post-PMJ phases may indicate that the chromospheric plasma is generally hotter than during the pre-PMJ stages. This is surprising because, due to the large chromospheric radiative losses, the dissipation timescales are expected to be considerably shorter than in the photosphere unless there is a continuous energy input. This aspect of PMJs should be investigated more thoroughly in the future.

Based on inversion results, previous works have reported an increase in the temperature associated with the PMJ brightening. Esteban Pozuelo et al. (2019) found that the PMJ region had a temperature 200-500 K larger than the surrounding environment above $\log (\tau)=-2$. This could be also true for the short-lived PMJs which at some places displayed intensity enhancements in the entire analyzed wavelength range. However, one would have to confirm that by performing non-LTE inversions of the Ca II $8542 \AA$ line.

Inversions are necessary to understand how the temperature, velocity, and magnetic field stratifications in the atmosphere evolve with time during the PMJs, but they require full Stokes observations in multiple lines while keeping a high temporal cadence. This is a particularly suitable task to be approached by the new generation of $4 \mathrm{~m}$ telescopes such as DKIST (Rimmele 2019) and EST (Collados et al. 2013), which are expected to provide unprecedented spectropolarimetric capabilities to overcome many of the current observational limitations.

Acknowledgements. We thank the referee for useful suggestions that greatly improved the paper. We also thank Sara Esteban Pozuelo for discussions and valuable comments. This work has been funded by the Spanish Ministry of Science and Innovation through project RTI2018-096886-B-C51, including a percentage from FEDER funds, and through the Centro de Excelencia Severo Ochoa grant SEV-2017-0709 awarded to the Instituto de Astrofísica de Andalucía in the period 2018-2022. The Swedish $1 \mathrm{~m}$ Solar Telescope is operated on the island of La Palma by the Institute for Solar Physics of Stockholm University in the Spanish Observatory del Roque de los Muchachos of the Instituto de Astrofísica de Canarias. The Institute for Solar Physics is supported by a grant for research infrastructures of national importance from the Swedish Research Council (registration number 2017-00625).

\section{References}

Bellot Rubio, L. R., Schlichenmaier, R., \& Tritschler, A. 2006, A\&A, 453, 1117 Borrero, J. M., \& Ichimoto, K. 2011, Liv. Rev. Sol. Phys., 8, 98

Buehler, D., Esteban Pozuelo, S., de la Cruz Rodríguez, J., \& Scharmer, G. B. 2019, ApJ, 876, 47

Cauzzi, G., Reardon, K. P., Uitenbroek, H., et al. 2008, A\&A, 480, 515

Cauzzi, G., Reardon, K., Rutten, R. J., Tritschler, A., \& Uitenbroek, H. 2009, A\&A, 503, 577

Centeno, R. 2018, ApJ, 866, 89

Collados, M., Bettonvil, F., Cavaller, L., \& EST Team 2013, in Highlights of Spanish Astrophysics VII, eds. J. Guirado, L. Lara, V. Quilis, \& J. Gorgas, Proceedings of the X Scientific Meeting of the Spanish Astronomical Society (SEA), held in Valencia, July 9-13, 2012, 808

de la Cruz Rodríguez, J., van der Rouppe Voort, L., Socas-Navarro, H., \& van Noort, M. 2013, A\&A, 556, A115

de la Cruz Rodríguez, J., Löfdahl, M. G., Sütterlin, P., Hillberg, T., \& van der Rouppe Voort, L. 2015, A\&A, 573, A40

Drews, A., \& Rouppe van der Voort, L. 2017, A\&A, 602, A80

Esteban Pozuelo, S., Bellot Rubio, L. R., \& de la Cruz Rodríguez, J. 2016, ApJ, 832,170

Esteban Pozuelo, S., de la Cruz Rodríguez, J., Drews, A., et al. 2019, ApJ, 870, 88

Fontela, J. M., Avrett, E. H., \& Loeser, R. 1993, ApJ, 406, 319

Grant, S. D. T., Jess, D. B., Zaqarashvili, T. V., et al. 2018, Nat. Phys., 14, 480

Hammar, J. 2014, Ph.D. Thesis, Uppsala Universitet, Sweden

Jurcák, J., \& Katsukawa, Y. 2008, A\&A, 488, L33

Jurcák, J., \& Katsukawa, Y. 2010, A\&A, 524, A21

Katsukawa, Y., \& Jurcăk, J. 2010, A\&A, 524, A20

Katsukawa, Y., Berger, T. E., Ichimoto, K., et al. 2007, Science, 318, 1594

Kosugi, T., Matsuzaki, K., Sakao, T., et al. 2007, Sol. Phys., 243, 3

Landi Degl'innocenti, E., \& Landolfi, M. 2004, Polarization in Spectral Lines (United States of America: Kluwer Academic Publishers)

Löfdahl, M. G. 2002, Proc. SPIE, 4792, 146

Magara, T. 2010, ApJ, 715, L40

Martínez González, M. J., \& Bellot Rubio, L. R. 2009, ApJ, 700, 1391

Quintero Noda, C., Shimizu, T., de la Cruz Rodríguez, J., et al. 2016, MNRAS, 459, 3363

Reardon, K., Tritschler, A., \& Katsukawa, Y. 2013, ApJ, 779, 143

Rempel, M., \& Schlichenmaier, R. 2011, Liv. Rev. Sol. Phys., 8, 3

Rimmele, T. 2019, BAAS, 51, 77

Ruiz Cobo, B., \& del Toro Iniesta, J. C. 1992, ApJ, 398, 375

Ryutova, M., Berger, T., Frank, Z., \& Title, A. 2008, ApJ, 686, 1404

Samanta, T., Tian, H., Banerjee, D., \& Schanche, N. 2017, ApJ, 835, L19

Scharmer, G. B., Bjelksjo, K., Korhonen, T. K., Lindberg, B., \& Petterson, B. 2003, SPIE Proc., 4853, 341

Scharmer, G. B., Narayan, G., Hillberg, T., et al. 2008, ApJ, 689, L69

Tiwari, S. K., Moore, R. L., Winebarger, A. R., \& Alpert, S. E. 2016, ApJ, 816, 92

Tiwari, S. K., Moore, R. L., De Pontieu, B., et al. 2018, ApJ, 869, 147

Tsuneta, S., Suematsu, Y., Ichimoto, K., et al. 2008, Sol. Phys., 249, 167

Uitenbroek, H. 2001, ApJ, 557, 389

van Noort, M., Rouppe van der Voort, L., \& Löfdahl, M. G. 2005, Sol. Phys., 228,191

Vissers, G. J. M., Rouppe van der Voort, L. H. M., \& Rutten, R. J. 2013, ApJ, 774,32

Vissers, G. J. M., Rouppe van der Voort, L. H. M., \& Carlsson, M. 2015, ApJ, 811, L33 